\title{
Shank-interacting protein-like 1 promotes tumorigenesis via PTEN inhibition in human tumor cells
}

\author{
Lizhi He,1,2,3 Alistair Ingram,1,2,3 Adrian P. Rybak, 1,2,3 and Damu Tang 1,2,3 \\ ${ }^{1}$ Division of Nephrology, Department of Medicine, McMaster University, ${ }^{2}$ Father Sean O'Sullivan Research Institute, and \\ ${ }^{3}$ Hamilton Centre for Kidney Research (HCKR), St. Joseph's Hospital, Hamilton, Ontario, Canada.
}

\begin{abstract}
Inactivation of phosphatase and tensin homolog (PTEN) is a critical step during tumorigenesis, and PTEN inactivation by genetic and epigenetic means has been well studied. There is also evidence suggesting that PTEN negative regulators (PTEN-NRs) have a role in PTEN inactivation during tumorigenesis, but their identity has remained elusive. Here we have identified shank-interacting protein-like 1 (SIPL1) as a PTEN-NR in human tumor cell lines and human primary cervical cancer cells. Ectopic SIPL1 expression protected human U87 glioma cells from PTEN-mediated growth inhibition and promoted the formation of HeLa cell-derived xenograft tumors in immunocompromised mice. Conversely, siRNA-mediated knockdown of SIPL1 expression inhibited the growth of both HeLa cells and DU145 human prostate carcinoma cells in vitro and in vivo in a xenograft tumor model. These inhibitions were reversed by concomitant knockdown of PTEN, demonstrating that SIPL1 affects tumorigenesis via inhibition of PTEN function. Mechanistically, SIPL1 was found to interact with PTEN through its ubiquitin-like domain (UBL), inhibiting the phosphatidylinositol 3,4,5-trisphosphate $\left(\mathrm{PIP}_{3}\right)$ phosphatase activity of PTEN. Furthermore, SIPL1 expression correlated with loss of PTEN function in PTEN-positive human primary cervical cancer tissue. Taken together, these observations indicate that SIPL1 is a PTEN-NR and that it facilitates tumorigenesis, at least in part, through its PTEN inhibitory function.
\end{abstract}

\section{Introduction}

While PTEN displays phosphatase activity for both protein and lipid substrates (1), accumulating evidence reveals that its lipid phosphatase activity, which dephosphorylates the 3 '-position phosphate from the inositol ring of phosphatidylinositol 3,4,5-triphosphate $\left(\mathrm{PIP}_{3}\right)(2,3)$, contributes to PTEN's tumor suppression activities. Thus, PTEN directly antagonizes a critical oncogenic activity mediated by PI3K $(4,5)$.

Consistent with its biochemical functions, inactivation of PTEN is a critical step during tumorigenesis. Typical mechanisms responsible for PTEN inactivation in human cancers include genetic and epigenetic events. The PTEN gene is frequently mutated in human cancers (6-8), including more than $50 \%$ of glioblastomas and melanomas, $30 \%-50 \%$ of endometrial carcinomas, and $10 \%$ of breast cancers $(4,9,10)$. A missense mutation, PTEN/G129E, leading to loss of PTEN's PIP 3 phosphatase activity $(4,11)$, was detected in Cowden disease (12), a multiple hamartoma syndrome with predisposition to multisystemic malignant tumors (13). Hypermethylation of the PTEN promoter in sporadic colorectal cancers and reduction of PTEN protein without mutations in the PTEN gene in prostate and cervical cancers have also been observed (14-16).

Inactivation of PTEN is known to contribute to cervical tumorigenesis. Low levels (2\%-16\%) of mutations in the PTEN gene as well as the loss of heterozygosity of PTEN have been reported in cervical cancer (17-20). Consistent with the hypermethylation of PTEN in cervical cancer, reduction in the PTEN protein was observed in approximately $15 \%$ of cervical squamous cell carcinomas $(21,22)$. Furthermore, essential pathways that promote cervical tumorigenesis have been shown to inhibit PTEN function. The E6 and E7

Conflict of interest: The authors have declared that no conflict of interest exists. Citation for this article: J Clin Invest. 2010;120(6):2094-2108. doi:10.1172/JCI40778. proteins of human papillomaviruses (HPVs) 16 and 18 are causal factors of cervical cancer $(23,24)$. While E6 has been well characterized to induce $\mathrm{p} 53$ degradation $(23,24)$, recent research revealed that E6 also activates the PI3K/AKT pathway by mediating PTEN degradation (25). Furthermore, a promising plant-derived cancerpreventing compound, indole-3-carbinol, inhibits cervical tumorigenesis by inducing PTEN expression (26). Although experimental evidence clearly demonstrated an important role for PTEN inactivation during cervical tumorigenesis, approximately $80 \%$ of squamous cell cervical carcinomas are PTEN positive $(21,22)$. This suggests that putative PTEN negative regulators (PTEN-NRs) may overcome PTEN's function during cervical tumorigenesis. Supporting this concept, amplification of the locus (PIK3CA) that encodes the P110 $\alpha$ catalytic subunit of class IA PI3K was observed in cervical cancer (27). Similar roles for PTEN inactivation during breast tumorigenesis have been well documented (28).

We report herein that PTEN is inactivated by a novel PTEN-NR during cervical tumorigenesis. Shank-interacting protein-like 1 (SIPL1; also known as Shank-associated PH domain interactor [SHARPIN]) binds to PTEN both in vitro and in vivo (primary cervical cancer), resulting in inhibition of PTEN's PIP ${ }_{3}$ phosphatase activity. We therefore demonstrate that PTEN is directly inactivated by an inhibitory protein during tumorigenesis of a primary human cancer.

\section{Results}

Identification of SIPL1 as a novel PTEN-NR2. Ectopic PTEN suppresses the proliferation of a variety of PTEN-null cancerous cells, including those of glioma, melanoma, and breast cancer $(2,8,29,30)$. Consistent with these reports, we observed that ectopic PTEN overexpression potently inhibited the proliferation of $\mathrm{LNCaP}$ prostate cancer cells among other cell lines tested, including PC3, DU145 
A

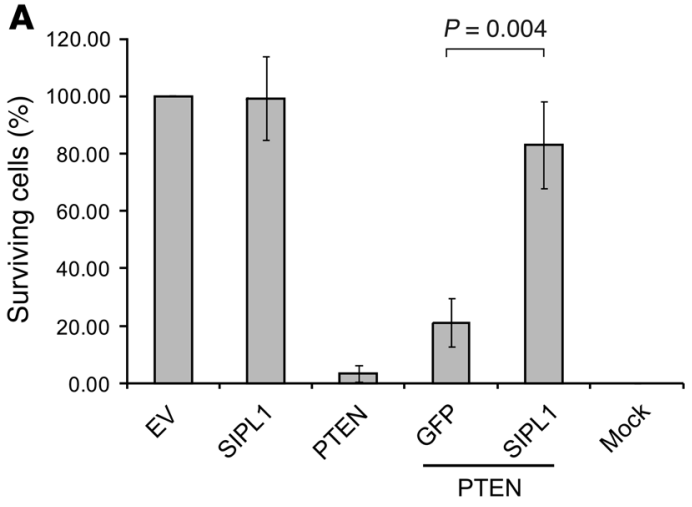

\section{C}

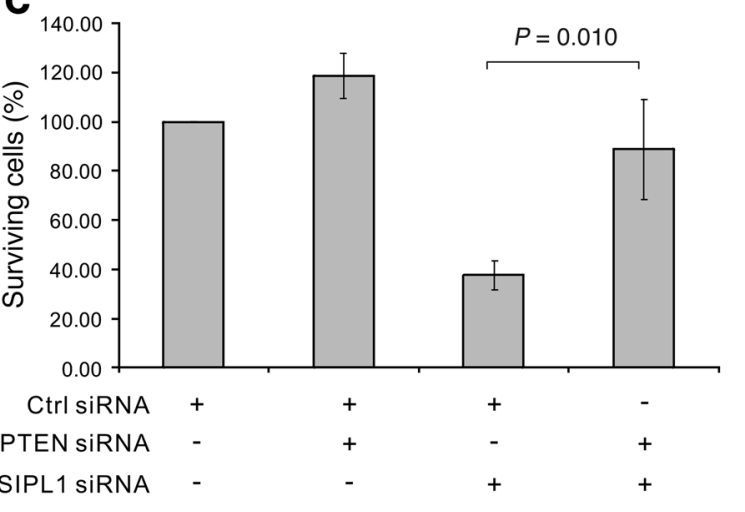

$\mathbf{E}_{1200}$

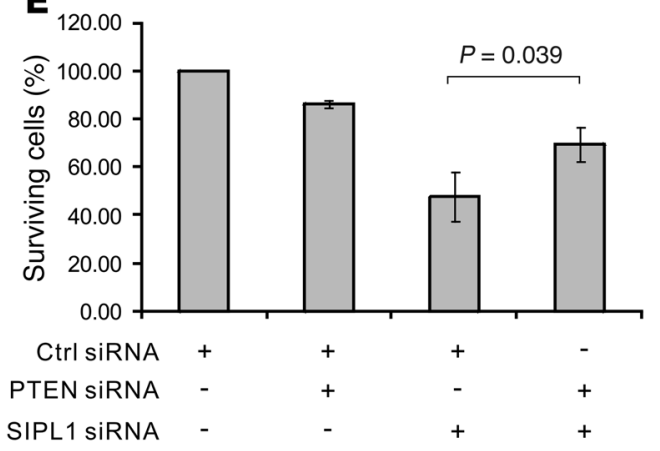

B

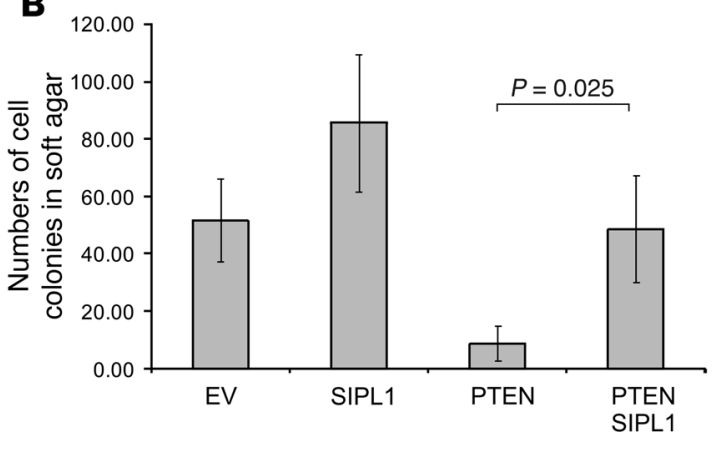

D

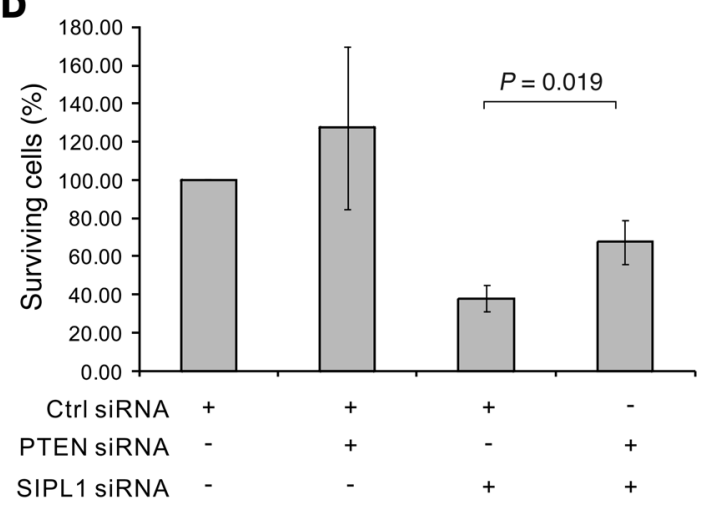

\section{Figure 1}

SIPL1 reduces PTEN function. (A) U87 cells were infected with retrovirus expressing EV, SIPL1, PTEN, PTEN-IRES-GFP, and PTEN-IRES-SIPL1. Expression of the respective proteins was demonstrated (Supplemental Figure 2A). Infected cells were cultured in hygromycin-containing medium for 2-3 weeks until surviving colonies formed, which were stained with crystal violet (Supplemental Figure 2A). Numbers of surviving cell colonies were quantified using ImageJ software and standardized as percentages of surviving EV cells. "Mock" indicates uninfected U87 cells were cultured in hygromycin-containing medium. Experiments were repeated twice. (B) U87 cells were infected with the indicated retrovirus. Cells $\left(2 \times 10^{4}\right)$ were then seeded in a 6-well soft agar plate and cultured for 4 weeks. Cell colonies were quantified. Experiments were repeated twice. (C) DU145, (D) $\mathrm{HeLa}$, and (E) HCC1954 cells were infected with retrovirus expressing a control (Ctrl) siRNA or SIPL1 siRNA, PTEN siRNA, or SIPL1/PTEN siRNA. Knockdown of individual proteins was demonstrated (see Figure 2A). Infected cells were selected in puromycin. Surviving cell colonies were stained (see Supplemental Figure 3A for typical images), and the number of surviving colonies was graphed. Experiments were repeated 3 times.

(Supplemental Figure 1A), U87, 293T, and NIH3T3 (data not shown). The potency of ectopic PTEN overexpression in inhibiting LNCaP cell growth enabled us to formulate a strategy to screen for PTEN-NRs (Supplemental Figure 1B). To develop this screen, we coexpressed PTEN together with an empty vector (EV) or a constitutively active AKT (AKT-DD) (31) in LNCaP cells. While ectopic AKT-DD induced no discernible adverse effect on LNCaP cell proliferation compared with the EV control, it was able to protect LNCaP cells to a certain extent from PTEN-mediated growth inhi- bition (Supplemental Figure 1C). PCR confirmed ectopic PTEN expression in the majority of surviving LNCaP cells coinfected with PTEN/AKT-DD (data not shown). These observations confirm the feasibility of using LNCaP cells to screen for PTEN-NRs.

To screen for PTEN-NRs, we coinfected LNCaP cells with pBabe-PTEN plus either a pLIB-based retroviral cDNA library derived from normal human prostate or an empty retrovirus (pLIB) (Supplemental Figure 1B). Coinfected cells were selected with puromycin (a selection marker specific for $\mathrm{pBabe-based}$ 


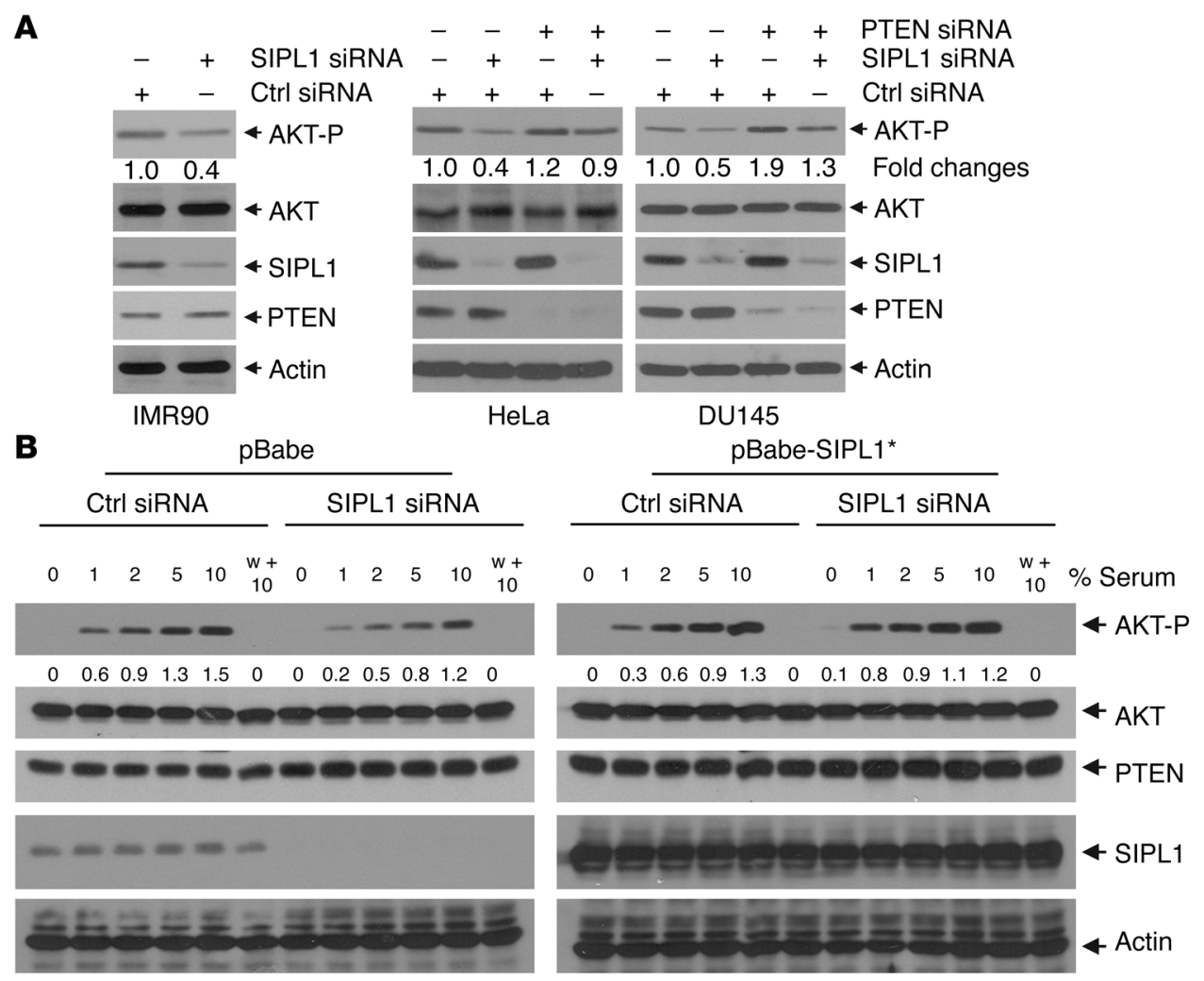

Figure 2

SIPL1 facilitates AKT activation by reducing PTEN function. (A) IMR90, HeLa, and DU145 cells were infected with the indicated retrovirus, followed by Western blot analysis for the phosphorylation of AKT Ser473 (AKT-P), AKT, SIPL1, PTEN, and actin using specific antibodies. The relative levels of AKT-P were normalized against the respective AKT and then expressed as fold change in AKT-P relative to that in Ctrl siRNA-treated cells. (B) DU145 cells were first infected with Ctrl siRNA or SIPL1 siRNA, followed by infection with pBabe or pBabe-SIPL1* (siRNA-resistant SIPL1). Infections were selected in media containing proper antibiotics to achieve $100 \%$ infection. Cells were then serum starved for 6 hours, stimulated with the indicated doses of serum for 90 minutes (according to the predetermined kinetics to achieve the plateau level of AKT activation; Supplemental Figure 5A), and analyzed for the indicated proteins by Western blot. Individual blots in the left and right panels, respectively, were derived from the same membranes. $w+10$, wortmannin (100 nM) plus $10 \%$ serum. AKT-P was quantified by first normalizing AKT against the respective actin and then normalizing AKT-P against the normalized total AKT. Levels of AKT-P are presented below the AKT-P panel. Quantification of AKT-P was performed using Scion Image software on scanned Western blot images. Experiments in $\mathbf{A}$ and $\mathbf{B}$ were repeated 3 times, and representative images are shown.

virus) for PTEN infection (Supplemental Figure 1B). Since $\mathrm{LNCaP}$ is a prostate cancer cell line, the normal prostate may contain PTEN-NRs. As expected, we recovered 38 surviving cell colonies containing human prostate cDNAs that were resistant to ectopic PTEN-mediated growth inhibition (data not shown). Only 4 surviving cell colonies were obtained from PTEN/pLIB coinfection (data not shown). PCR amplification of genomic DNA isolated from these colonies confirmed the existence of ectopic PTEN and resulted in the identification of 4 initial PTEN-NR candidates: $\alpha$-mannosidase 2C1 (MAN2C1), 3-hydroxysteroid epimerase (RODH), PTEN-NR1 (a hypothetical protein), and SIPL1 (PTEN-NR2) (Supplemental Figure 1D). The inability to recover known PTEN-NRs such as AKT may be attributed to the fact that they are not constitutively active in normal prostate. The fact that our system only identified a limited number of PTEN-NR candidates may be attributable to the low efficiency of the PCR-based methodology to recover ectopic cDNAs (see Supplemental Methods). Using LNCaP and U87 PTEN-null cells and following the strategy outlined in Supplemental Figure 1B, we subsequently confirmed that RODH showed no detectable activity in the attenuation of PTEN function (data not shown) and that MAN2C1 as well as PTEN-NR1 reduced PTEN function (data not shown).

SIPL1, which we initially called PTEN-NR2, contains a ubiquitin-like (UBL) domain between residues 240 and 300 (Supplemental Figure 1D) and was detected in multiple tissues (32). Although SIPL1 has been shown to associate with SHANK in the postsynaptic density of synapses and may play a role in enteric neural transmission $(32,33)$, the function of SIPL1 remains largely to be determined. To investigate SIPL1 function, we produced bacterial recombinant 6xHis-SIPL1 and GST-SIPL1 proteins (data not shown), which were used to raise an anti-SIPL1 antibody (see Methods for details). The antibody recognized endogenous SIPL1 in DU145 cells, which was specifically blocked by GST-SIPL1 but not GST (Supplemental Figure 1E, left panel). Knockdown of SIPL1 in DU145 cells substantially reduced the signal recognized by anti-SIPL1 antibody (Supplemental Figure 1E, right panel). The antibody also specifically recognized SIPL1 by immunofluorescence and immunohistochemistry (IHC) (data not shown). 
A

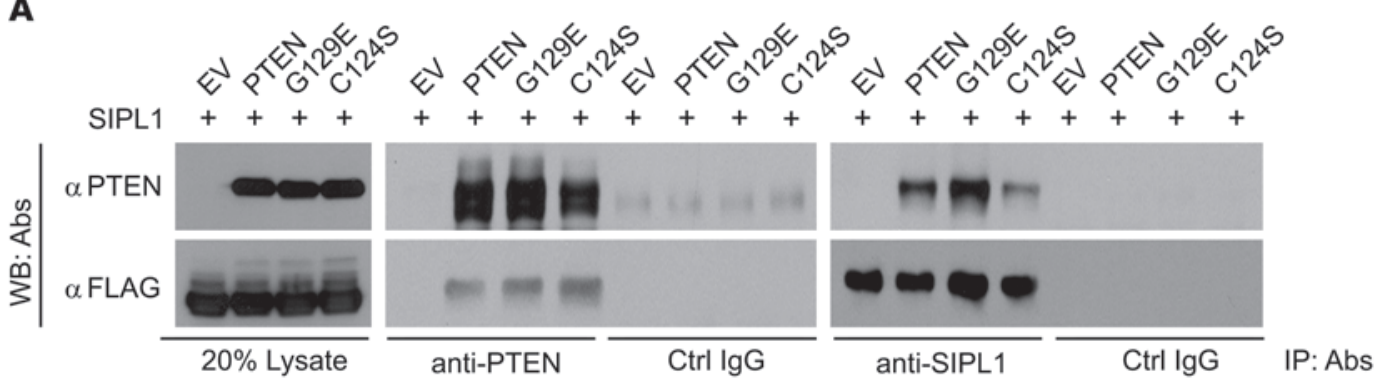

B
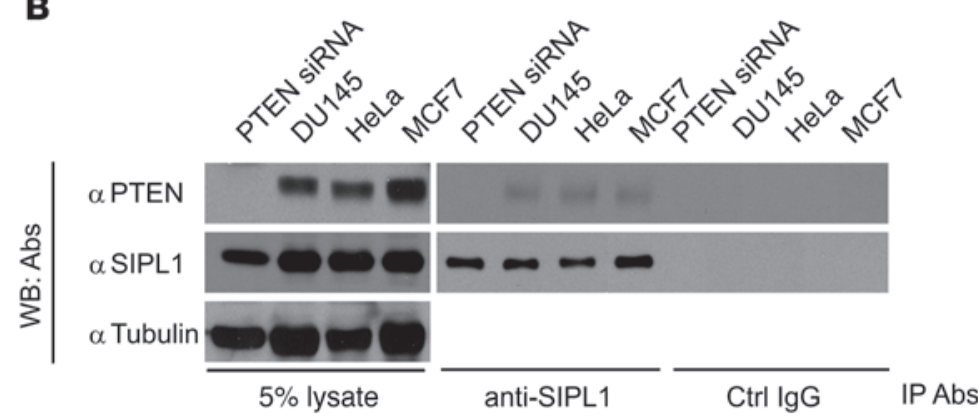

C
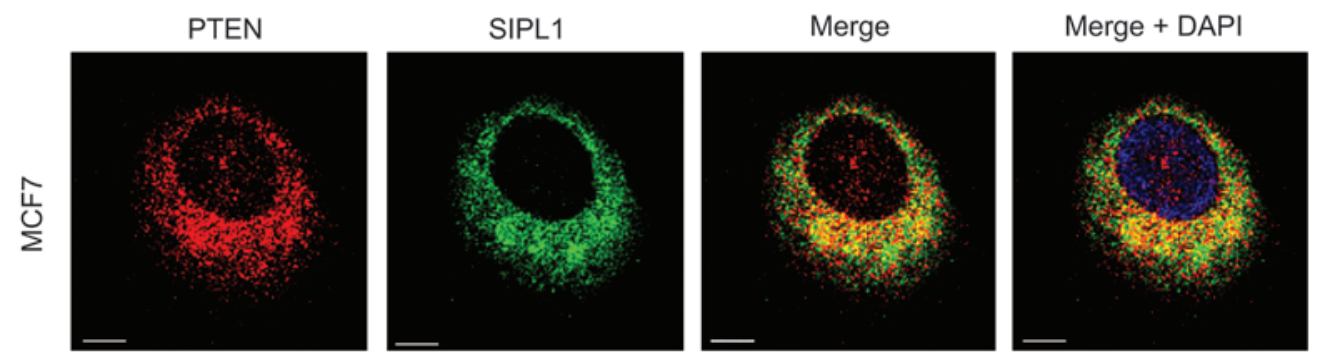

\section{Figure 3}

SIPL1 binds to PTEN. (A) SIPL1 was coexpressed with the indicated cDNAs in 293T cells, followed by IP with the indicated antibodies and Western blot (WB) analysis using anti-PTEN or anti-FLAG antibodies. (B) IP of endogenous SIPL1 with the indicated antibodies, followed by Western blot analysis for PTEN and SIPL1 using specific antibodies. "PTEN siRNA" indicates PTEN-knockdown DU145 cells. (C) Colocalization of PTEN and SIPL1 in MCF7 cells. PTEN and SIPL1 were immunofluorescently stained as red and green, respectively. Typical z-stack images captured using a confocal microscope (MP Leica TCS SP5) are shown. Nuclei were counterstained with DAPI (blue). Scale bars: $10 \mu \mathrm{m}$. Experiments in $\mathbf{A}, \mathbf{B}$, and $\mathbf{C}$ were repeated 3 times.

To examine whether SIPL1 attenuates PTEN function, we infected U87 cells with an EV retrovirus or retrovirus expressing SIPL1, PTEN, PTEN-IRES-GFP, or PTEN-IRES-SIPL1 (IRES, internal ribosome entry site) (Supplemental Figure 2A, left panel). As expected, ectopic PTEN reduced the number of surviving cell colonies formed, and ectopic SIPL1 enhanced U87 cell survival when PTEN was overexpressed (Figure 1A and Supplemental Figure 2A, right panel). The same observations were made when HA-tagged and non-tagged ectopic PTEN was used and when PTEN and SIPL1 were expressed from individual vectors. This was performed by establishing U87 EV and U87 SIPL1 cells first, followed by infection with PTEN or EV retrovirus (data not shown). Furthermore, when PTEN-IRES-GFP was expressed in U87 EV and U87 SIPL1 (stably expressing SIPL1) cells, morphological changes (indicative of apoptotic cell death) in U87 EV cells were clearly detected as early as 1 day after infection, while PTENIRES-GFP-infected U87 SIPL1 cells maintained viability even at day 5 after infection (Supplemental Figure 2B). In comparison to GFP retrovirus-infected U87 EV cells (approximately 90\% of U87 EV and U87 SIPL1 cells were GFP positive using either GFP or
PTEN-IRES-GFP retrovirus at 24 hours after infection), less than $10 \%$ of U87 EV cells infected with PTEN-IRES-GFP remained GFP positive at day 5 after infection (the majority of them being rounded up, indicative of apoptotic cell death), and 50\%-60\% of U87 SIPL1 cells infected with PTEN-IRES-GFP were healthy and GFP positive (data not shown). Similar results were obtained with and without using antibiotic selection (data not shown). Therefore, SIPL1-mediated attenuation of PTEN function was not due to the presence of the HA tag or using antibiotic selection, nor was it due to the expression of SIPL1 together with PTEN from a bicistronic transcript using IRES. Similar results were obtained when LNCaP cells were used (data not shown). Additionally, while PTEN overexpression inhibited U87 cell growth in soft agar, ectopic SIPL1 enabled U87 cells to form colonies in soft agar in the presence of PTEN overexpression (Figure 1B). Although overexpression of SIPL1 slightly enhanced U87 cell growth in soft agar (Figure 1B), which suggests that SIPL1 may enhance anchorageindependent cell growth independently of PTEN (see Discussion for details), the observation that ectopic SIPL1 overexpression significantly reversed ectopic PTEN overexpression-mediated 
A

\begin{tabular}{|c|c|c|c|c|c|c|c|c|}
\hline \multirow[b]{2}{*}{ EV } & \multicolumn{2}{|c|}{$10 \%$ lysate } & \multicolumn{3}{|c|}{$\alpha-F L A G(M 2)$} & \multicolumn{3}{|c|}{$\lg G$} \\
\hline & - & - & + & - & - & + & - & - \\
\hline PTEN & + & + & + & + & + & + & + & + \\
\hline PL1-C & 一 & + & - & + & 一 & - & + & - \\
\hline IPL1-N & + & - & - & - & + & - & - & + \\
\hline
\end{tabular}

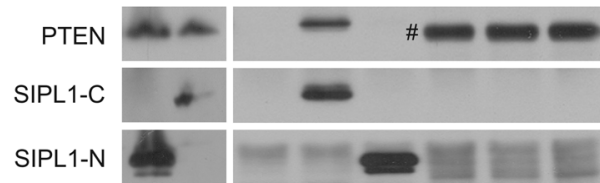

C

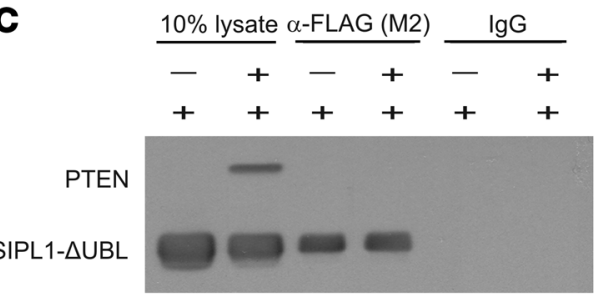

IPAb

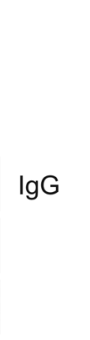

SIPLI-A

SIPL1- $\triangle$ UBL

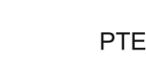

B

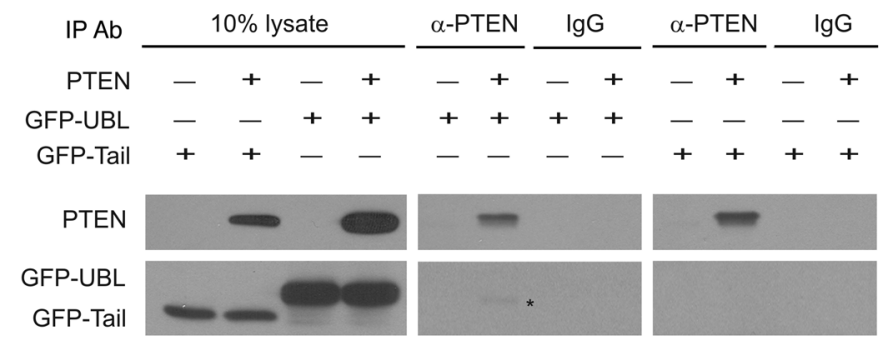

SIPL1- $\triangle$ UBL

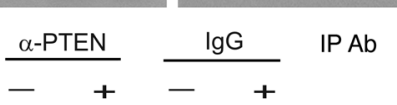

\begin{tabular}{llllll}
$10 \%$ lysate & \multicolumn{2}{l}{$\alpha$-PTEN } & \multicolumn{2}{l|}{ IgG } \\
\cline { 5 - 6 } & + & - & + & - & + \\
+ & + & + & + & + & +
\end{tabular}

PTEN
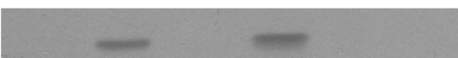
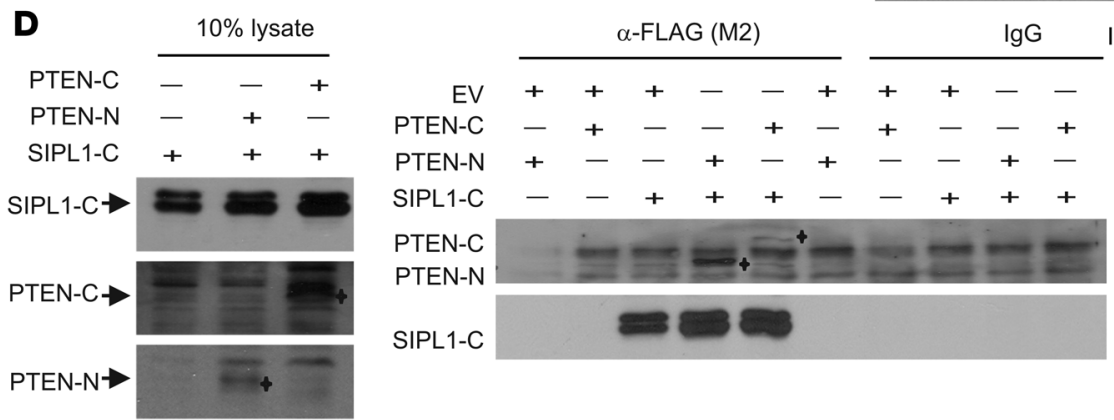

IP Ab

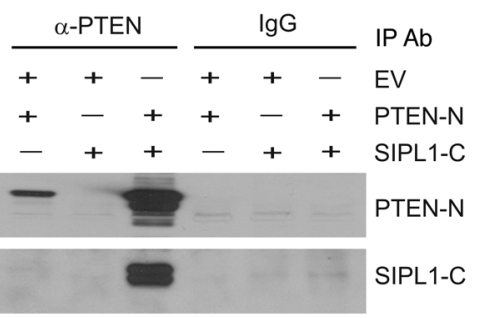

E

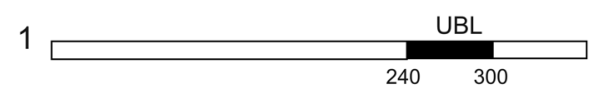

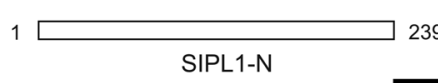
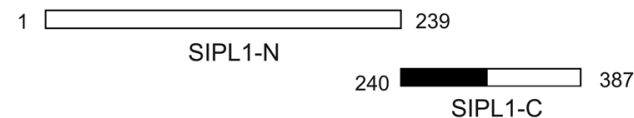

240

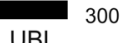

SLIP1- $\triangle U B L$

\section{1}

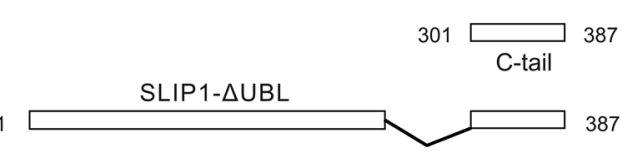

Binding to PTEN 387

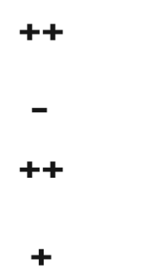

$-$

\section{Figure 4}

Characterization of the interaction between SIPL1 and PTEN. Truncation mutants of SIPL1 and PTEN used in the binding experiments were generated (E). (A) SIPL1-N and SIPL1-C mutants were coexpressed individually with PTEN in 293T cells, followed by IP with the indicated antibodies, and examined for PTEN, SIPL1-N, and SIPL1-C by Western blot. The pound symbol (\#) indicates the IgG heavy chain. (B) SIPL1 tail (see $\mathbf{E}$ for details) and UBL were fused with GFP (GFP-tail and GFP-UBL), followed by examination of their interaction with PTEN by transient transfection using 293T cells. The asterisk indicates the coimmunoprecipitated GFP-UBL band. (C) FLAG-tagged SIPL1-AUBL was examined for interaction with PTEN using the IP-Western blot methodology. (D) PTEN-N and PTEN-C were examined for binding to FLAG-tagged SIPL1-C by the IP-Western blot system using the indicated antibodies. PTEN-N and PTEN-C bands are indicated (left and middle panels). (E) Mapping the PTEN-binding and SIPL1-binding motifs.,,$++++/-$, and - indicate the levels of interaction between the indicated proteins.

inhibition of U87 cell growth in soft agar (Figure 1B) supports the concept that SIPL1 promotes anchorage-independent cell growth, at least in part, by reducing PTEN function. To further demonstrate this concept, we were able to show that knockdown of SIPL1 significantly inhibited the growth of multiple PTENpositive cell lines, including DU145, HeLa, and HCC1954 cells, by enhancing endogenous PTEN function, as co-knockdown of PTEN largely (DU145 cells) or partially (HeLa and HCC1954 cells) reversed the growth inhibition imposed by SIPL1 siRNA (Figure 1, C-E, and Supplemental Figure 3A). The specificity of our siRNA approach was confirmed by using multiple siRNAs (see Methods for details). We further excluded the possibility that 

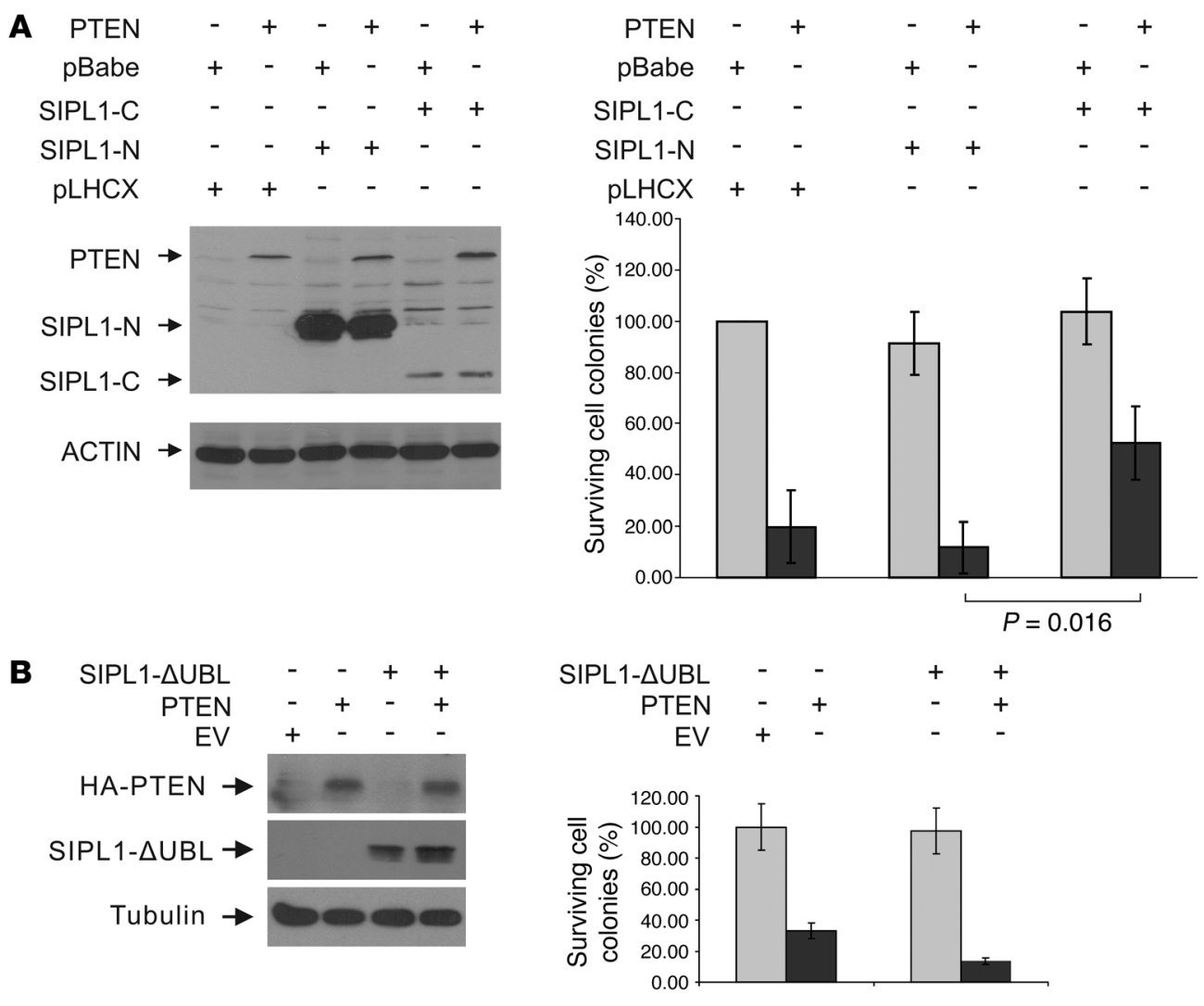

Figure 5

SIPL1 reduces PTEN function via binding to PTEN. U87 cells were infected with pLHCX(SIPL1-N and SIPL1-C), pBabebased (PTEN), or SIPL1- $\triangle \mathrm{UBL}$ retrovirus as indicated. Expression of individual proteins was determined (left panels, A and B). Surviving cells were stained with crystal violet (data not shown) and quantified (right panels, $\mathbf{A}$ and $\mathbf{B}$ ). Experiments were repeated twice.
SIPL1 siRNA may nonspecifically target other proteins, which might cause the aforementioned growth inhibition: (a) Knockdown of SIPL1 reduced the number of surviving cell colonies in DU145, 293T, HeLa, and MCF7 cells but did not affect the growth of BPH-1 (an SV40 immortalized prostate hyperplasia epithelial cell line) (data not shown). Although BPH-1 cells express a readily detectable SIPL1 (data not shown), their insensitive status to SIPL1 knockdown may be attributable to their nonmalignant status. (b) When an siRNA-resistant SIPL1*, which was produced by changing cDNA sequences targeted by the siRNA but maintaining amino acid identities, was ectopically expressed in $\mathrm{HeLa}$ cells (Supplemental Figure 3B, top panel), SIPL1* significantly protected HeLa cells from the growth inhibition imposed by SIPL1 siRNA (Supplemental Figure 3B, bottom panel, and Supplemental Figure 3C). To further support SIPL1's ability to affect cell proliferation, at least in part, by inhibiting PTEN function, we ectopically expressed SIPL1 in multiple PTEN-negative U87, C33A (see Methods for the PTEN-negative status of C33A cells), and LNCaP cells (data not shown). Ectopic SIPL1 did not affect the proliferation of these cells (Supplemental Figure 4, A and B). Furthermore, knockdown of SIPL1 in U87 and LNCaP cells did not alter their proliferation (Supplemental Figure 4, A and C). Taken together, the above observations demonstrate that SIPL1 attenuates PTEN function.

To further support this concept, we demonstrated that knockdown of SIPL1 reduced AKT activation by 50\%-60\% in IMR90, HeLa, DU145 (Figure 2A), and MCF7 cells (data not shown) and concomitant knockdown of PTEN reversed SIPL1 siRNA-mediated reduction of AKT activation in HeLa and DU145 cells (Figure 2A), demonstrating that knockdown of SIPL1 attenuated
AKT activation by enhancing endogenous PTEN function. Furthermore, we were able to show that SIPL1 plays a role in seruminduced activation of the PI3K/AKT pathway. To examine this issue, we first optimized serum-induced AKT activation in DU145 cells (Supplemental Figure 5A). Using optimized conditions, we found that knockdown of SIPL1 in DU145 cells attenuated serum-induced activation of the PI3K/AKT pathway, especially at low doses of serum (Figure 2B). This attenuation was prevented by introducing an siRNA-resistant SIPL1* (Figure 2B). Furthermore, using the PI3K inhibitor wortmannin, we confirmed that serum-induced AKT-P depends on PI3K (Figure 2B). Additionally, ectopic SIPL1 sensitizes serum-induced AKT activation in MCF7 cells (Supplemental Figure 5B). Taken together, our results demonstrate that SIPL1 negatively regulates PTEN function.

SIPL1 binds to PTEN. To determine the underlying mechanism whereby SIPL1 reduces PTEN function, we examined a possible interaction between SIPL1 and PTEN. FLAG-tagged SIPL1 was expressed with or without PTEN in 293T cells. IP of PTEN precipitated SIPL1 and vice versa, while a control IgG did not precipitate either SIPL1 or PTEN (Figure 3A). This interaction did not require PTEN's phosphatase activity, as the phosphatase-deficient PTEN (C124S) mutant and the $\mathrm{PIP}_{3}$ phosphatase-deficient PTEN (G129E) mutant (11) formed complexes with SIPL1 (Figure 3A). Furthermore, endogenous SIPL1 and endogenous PTEN were also coimmunoprecipitated from DU145, MCF7, and HeLa cells (Figure 3B). To further demonstrate the endogenous SIPL1/PTEN complex being coimmunoprecipitated, we knocked down PTEN in DU145 cells, which prevented the coimmunoprecipitation of PTEN via SIPL1 (Figure 3B). In the cell, ectopic SIPL1 colocalized with ectopic PTEN in 293T (Supplemental Figure 6A) and U87 cells 
A

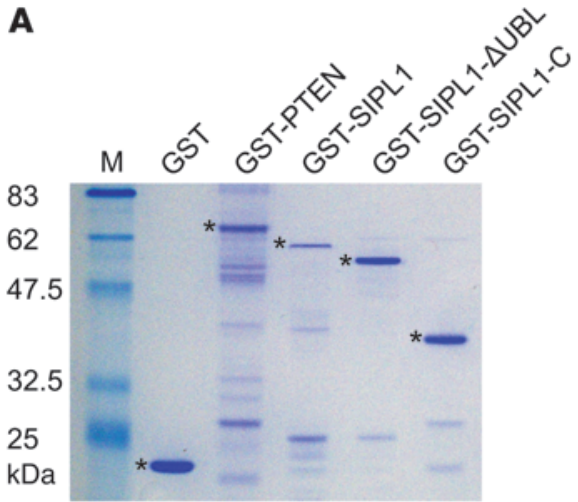

C

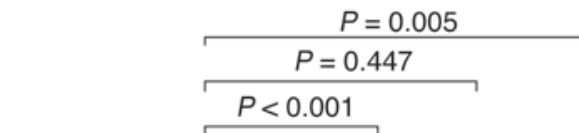

B

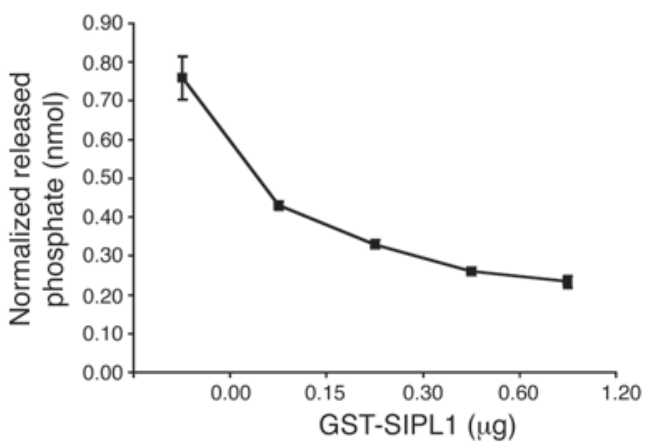

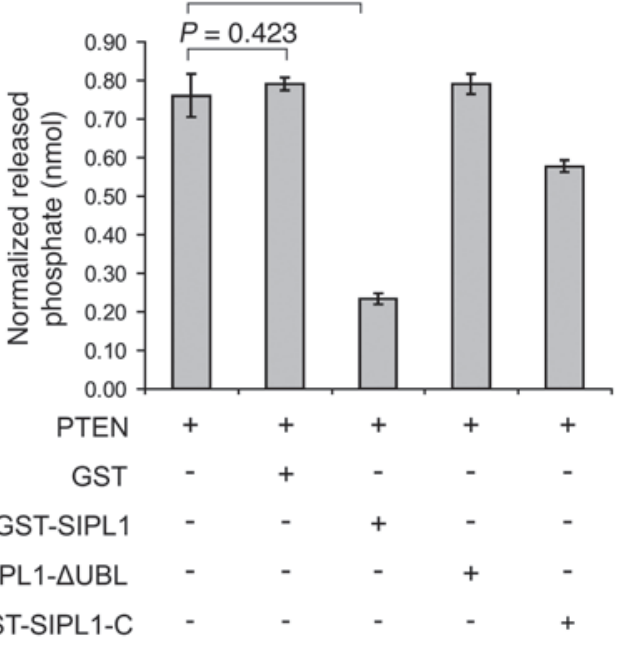

Figure 6

SIPL1 inhibits PTEN's PIP 3 phosphatase activity. (A) The indicated recombinant GST fusion proteins were purified from E. coli (BL21). The asterisks indicate the respective recombinant proteins. (B) GST-PTEN $(0.3 \mu \mathrm{g})$ was incubated with the indicated doses of GST-SIPL1 on ice for 30 minutes, followed by assaying for PTEN's PIP ${ }_{3}$ phosphatase activity. (C) GST-PTEN $(0.3 \mu \mathrm{g})$ was incubated with $1.2 \mu \mathrm{g}$ of the indicated GST fusion proteins, followed by determination of PTEN's $\mathrm{PIP}_{3}$ phosphatase activity.

(data not shown). Endogenous PTEN colocalized with endogenous SIPL1 in MCF7, HeLa, HCC1954, and DU145 cells (Figure 3C and Supplemental Figure 6B). This colocalization took place exclusively in the cytosol (Figure 3C, Supplemental Video 1, and Supplemental Figure 6). This result is in agreement with SIPL1 being a cytosolic protein $(32,33)$. Taken together, the above observations demonstrate an association between SIPL1 and PTEN.

We subsequently mapped the domains involved in the interaction between PTEN and SIPL1. A set of SIPL1 truncation mutants was generated (Figure 4E) and examined for their association with PTEN. When coexpressed in 293T cells, SIPL1-C but not SIPL1-N coimmunoprecipitated PTEN (Figure 4A). Further mapping of the PTEN-binding motif in SIPL1-C revealed that the UBL is capable of interacting with PTEN (Figure 4, B and $\mathrm{E}$ ). Although the $\mathrm{C}$-terminal tail region did not associate with PTEN (Figure 4, B and E), it facilitated the interaction between SIPL1-C and PTEN, as coimmunoprecipitation of the SIPL1-C/PTEN complex was much more efficient than coimmunoprecipitation of the UBL/PTEN complex (Figure 4, A and B).
Furthermore, deletion of the UBL domain rendered SIPL1 incapable of binding to PTEN (Figure 4, C and E). These results are consistent with our identification of the C-terminal SIPL1 fragment encompassing residues 225-387 (see Supplemental Methods for details) in our initial screen, which contains SIPL1-C (residues 240-387; Figure 4E).

We further investigated the SIPL1-binding motifs of PTEN. An $\mathrm{N}$-terminal fragment (PTEN-N) containing the catalytic domain and a C-terminal fragment (PTEN-C) excluding the catalytic domain were constructed (Figure 4E). When coexpressed with SIPL1-C in 293T cells, PTEN-N was readily coprecipitated by SIPL1-C, while PTEN-C was rather weakly coprecipitated via SIPL1-C (Figure 4D). SIPL1-C was also very efficiently coprecipitated by PTEN-N (Figure 4D). Taken together, these observations reveal that PTEN's catalytic fragment (PTEN-N) binds to SIPL1 (Figure 4E).

SIPL1 reduces PTEN function via binding to PTEN. To demonstrate that SIPL1 attenuates PTEN function through its physical association with PTEN, we determined the impact of SIPL1 mutants on PTEN-mediated inhibition of U87 cell growth. When coex- 

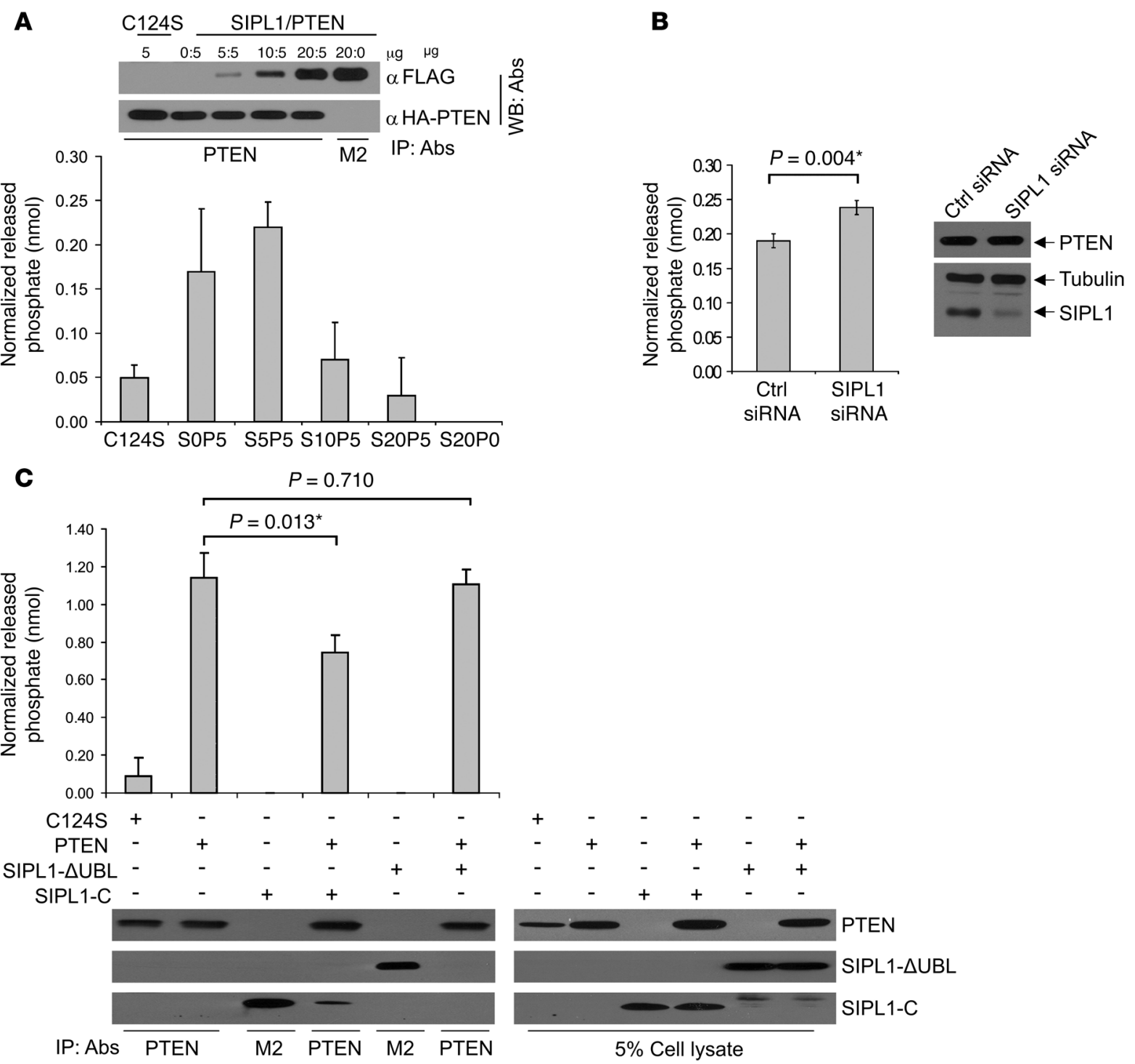

\section{Figure 7}

SIPL1 reduces PTEN's PIP 3 phosphatase activity in the cell. (A) 0, 5, 10, $20 \mu \mathrm{g}$ SIPL1 and $5 \mu \mathrm{g}$ PTEN (S0P5, S5P5, S10P5 and S20P5, where $S$ represents SIPL1) were cotransfected into 293 T cells. $5 \mu \mathrm{g}$ PTEN C124S mutant (C124S) served as a negative control. IPs were carried out using an anti-PTEN antibody, followed by assaying for PTEN's PIP 3 phosphatase activity. S20P0, $20 \mu$ g SIPL1 (FLAG-tagged) was used (negative control). Other negative controls (a reaction without the addition of PTEN and a reaction without the addition of PIP $\mathrm{P}_{3}$ substrate) showed no detectable activities (data not shown). Western blot shows the immunoprecipitated PTEN and SIPL1. (B) DU145 cells were treated with control siRNA or SIPL1 siRNA retrovirus as indicated. Knockdown of SIPL1 was demonstrated (right panel). PTEN was immunoprecipitated from both cells, followed by assaying for PTEN's $\mathrm{PIP}_{3}$ phosphatase activity in vitro. Comparable levels of PTEN were immunoprecipitated from both cell lines (data not shown). Experiments were repeated 3 times. (C) PTEN (5 $\mu$ g) was transiently coexpressed in 293 T cells with the indicated SIPL1 mutants $(20 \mu \mathrm{g})$, followed by IP with anti-PTEN or M2 anti-FLAG (for SIPL1 mutants), and then assayed for PTEN's PIP 3 phosphatase activity. PTEN C124S (C124S, $5 \mu \mathrm{g}$ ) was used as a negative control. Under this assay system, $20 \mu \mathrm{g}$ of the SIPL1 construct completely inhibited the PIP 3 phosphatase activity produced by cotransfection of cells with $5 \mu \mathrm{g}$ of the PTEN construct (data not shown; also see A).

pressed in U87 cells, SIPL1-C but not SIPL1-N enhanced U87 cell survival in the presence of PTEN overexpression (Figure 5A). This is consistent with the observations (Figure 4E) that SIPL1-C but not SIPL1-N interacts with PTEN and that the C-terminal SIPL1 fragment consisting of residues 225-387, which contains SIPL1-C (encompassing residues 240-387), was identified in our screen for its ability to reduce PTEN function (Supplemental Methods). To further support this observation, we specifically deleted the UBL domain from SIPL1 (SIPL1- $\triangle U B L)$. SIPL1- $\triangle U B L$ was incapable of associating with PTEN (Figure 4E) and was unable to protect U87 cells from PTEN overexpression-mediated growth inhibition (Figure 5B).

SIPL1 inhibits PTEN's PIP 3 phosphatase activity. Our observations that SIPL1 reduces PTEN function (Figures 1 and 2) and binds to PTEN's catalytic fragment (Figure 4E) prompted us to determine the impact of this interaction on PTEN's $\mathrm{PIP}_{3}$ phosphatase activity. To address this issue, we generated a set of recombinant proteins in E. coli: GST, GST-PTEN, GST-SIPL1, GST-SIPL1-C, and 


\section{Table 1}

SIPL1 promotes DU145 cell-derived xenograft tumor formation

$\begin{array}{lcccc}\text { Days }^{A} & \begin{array}{c}\text { Cell } \\ \text { lines }\end{array} & \begin{array}{c}\text { Tumor volume }\left(\mathbf{m m}^{3}\right), \\ \text { mean } \pm \text { SEM }\end{array} & \begin{array}{c}\text { Tumor } \\ \text { incidence }\end{array} & \begin{array}{c}\text { Mann-Whitney } \\ \text { U test }\end{array} \\ 66 & \text { EV } & 40.21 \pm 22.04 & 5 / 10 & \\ & \text { SIPL1 } & 121.69 \pm 43.30 & 7 / 10 & 0.197 \\ 73 & \text { EV } & 45.20 \pm 23.44 & 5 / 10 & \\ & \text { SIPL1 } & 125.24 \pm 44.32 & 7 / 10 & 0.197 \\ 80 & \text { EV } & 23.32 \pm 13.53 & 4 / 10 & \\ & \text { SIPL1 } & 158.67 \pm 48.77 & 8 / 10 & 0.019\end{array}$

ADays after tumor implantation. BDU145 cells stably transfected with either EV or SIPL1. CNumber of xenograft tumors formed/number of implantations. Five nude mice were used for each cell line. Both side flanks were implanted.

GST-SIPL1- $\triangle$ UBL (Figure 6A). While GST-SIPL1 dose-dependently inhibited the $\mathrm{PIP}_{3}$ phosphatase activity of GST-PTEN (Figure 6B), GST had no effect on GST-PTEN's $\mathrm{PIP}_{3}$ phosphatase activity when used at the highest level (Figure 6C). Consistent with SIPL1- $\triangle U B L$ being incapable of binding to PTEN (Figure 4E), GST-SIPL1$\triangle$ UBL did not inhibit GST-PTEN's PIP 3 phosphatase activity (Figure 6C). While GST-SIPL1-C reduced recombinant PTEN's
$\mathrm{PIP}_{3}$ phosphatase activity, which is consistent with its ability to associate with PTEN (Figure 4E), GST-SIPL1-C was much less efficient than GST-SIPL1 at inhibiting GST-PTEN's PIP 3 phosphatase activity (Figure 6C), suggesting that SIPL1-N may play a role in SIPL1-mediated inhibition of PTEN's $\mathrm{PIP}_{3}$ phosphatase activity.

To support the above observations, we demonstrated that SIPL1 also reduces PTEN's $\mathrm{PIP}_{3}$ phosphatase activity in the cell. When phosphatase-deficient PTEN(C124S) (11), PTEN, SIPL1, and PTEN plus SIPL1 were transiently expressed in 293T cells, IP of PTEN dose-dependently coprecipitated SIPL1 (Figure 7A). While immunoprecipitated PTEN dephosphorylated PIP $_{3}$ in vitro, PTEN(C124S) displayed no such activity, as expected (Figure 7A). When complexed with PTEN, SIPL1 dose-dependently reduced PTEN's $\mathrm{PIP}_{3}$ phosphatase activity (Figure 7A). It is possible that SIPL1 did not reduce PTEN's $\mathrm{PIP}_{3}$ phosphatase activity at the ratio of 5:5 (SIPL1/PTEN) (Figure 7A) because there was an insufficient amount of SIPL1 protein available to interact with PTEN. The fact that at a ratio of 20:5 (SIPL1/PTEN), SIPL1 reduced PTEN's PIP 3 phosphatase activity to a level comparable to PTEN(C124S)-associated background activity shows that SIPL1 is able to completely inhibit PTEN's $\mathrm{PIP}_{3}$ phosphatase activity at least in vitro (Figure 7A). To further address SIPL1-mediated inactivation of PTEN's PIP 3 phosphatase activity, we were able to knock down SIPL1 in DU145 cells and observed a significant increase in PTEN's PIP 3
A

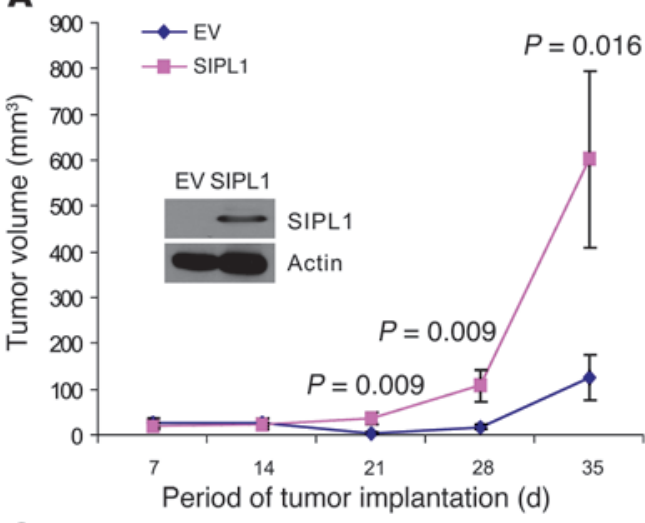

C

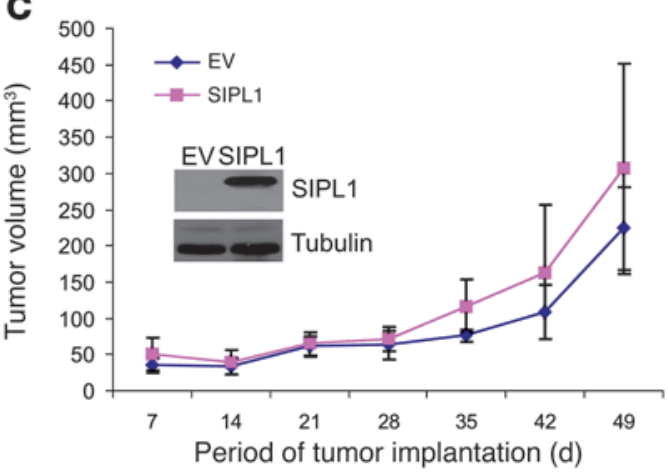

B

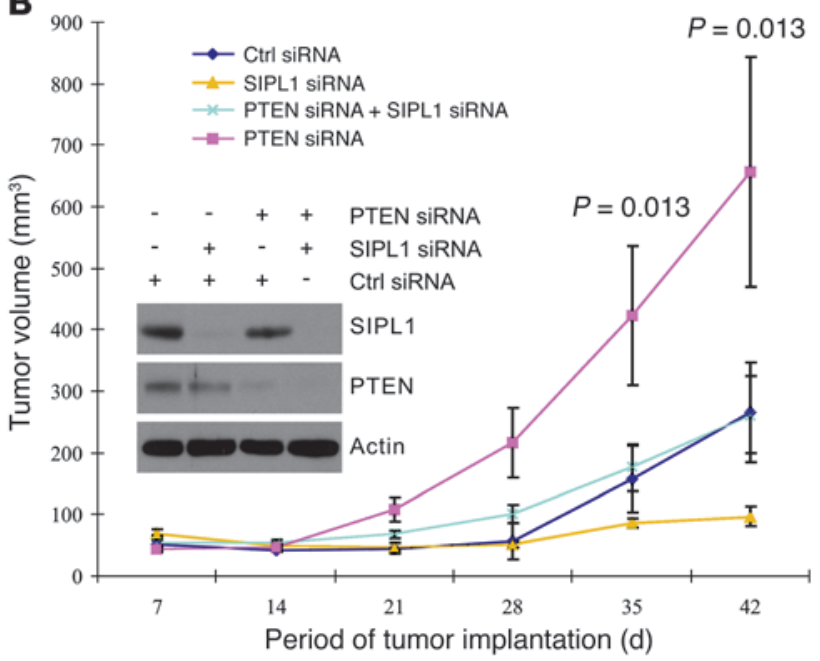

Figure 8

SIPL1 promotes xenograft tumor formation by inhibiting PTEN function. PTEN-positive cervical cancer HeLa (A) and PTEN-negative cervical cancer C33A (C) cells were infected with the indicated retrovirus. Expression of ectopic SIPL1 was confirmed (insets). Cells (106) were s.c. implanted into NOD/SCID mice (5 mice per group). Tumor volumes were measured and graphed. Tumor incidence for $\mathbf{A}$ and $\mathbf{C}$ is 5 of 5 (number of tumors formed/ number of implantations) for the time points of 21 days onward. IHC staining confirmed ectopic SIPL1 in xenograft tumors from SIPL1-expressing C33A cells (data not shown). (B) HeLa cells were treated with the indicated retroviruses. Knockdown of individual proteins was confirmed (inset). Cells $\left(1.5 \times 10^{5}\right)$ were implanted into NOD/SCID mice $(5$ mice per group). Tumor volumes were monitored weekly and graphed. The $P$ values were derived from statistical analyses made among the 4 groups, as determined by a 1-way ANOVA. Data in $\mathbf{A}-\mathbf{C}$ are presented as mean \pm SEM. 


\begin{tabular}{|c|c|c|c|c|}
\hline Days & $\begin{array}{l}\text { siRNA } \\
\text { treatment }{ }^{B}\end{array}$ & $\begin{array}{c}\text { Tumor volume }\left(\mathrm{mm}^{3}\right) \text {, } \\
\text { mean } \pm \text { SEM }\end{array}$ & $\begin{array}{c}\text { Tumor } \\
\text { incidence }\end{array}$ & $\begin{array}{c}\text { Mann-Whitney } \\
\text { U test }\end{array}$ \\
\hline \multirow[t]{2}{*}{12} & Ctrl siRNA & $33.64 \pm 9.00$ & $7 / 7$ & \\
\hline & SIPL1 siRNA & $2.23 \pm 1.29$ & $3 / 7$ & 0.030 \\
\hline \multirow[t]{2}{*}{15} & Ctrl siRNA & $63.59 \pm 40.66$ & $7 / 7$ & \\
\hline & SIPL1 siRNA & $5.38 \pm 3.46$ & $3 / 7$ & 0.017 \\
\hline \multirow[t]{2}{*}{18} & Ctrl siRNA & $139.48 \pm 91.23$ & $7 / 7$ & \\
\hline & SIPL1 siRNA & $16.08 \pm 10.43$ & $3 / 7$ & 0.043 \\
\hline
\end{tabular}

ADays after the delivery of siRNA directly into xenograft tumors. ${ }^{\mathrm{B}} \mathrm{HeLa}$ cells were s.c. implanted into NOD/SCID mice; at day 7, control (Ctrl) siRNA or SIPL1 SiRNA was directly delivered into tumors. CNumber of xenograft tumors formed/number of implantations. Five NOD/SCID mice were used for each siRNA treatment. Two mice were s.c. implanted on both side flanks.

phosphatase activity when compared with PTEN's PIP $_{3}$ phosphatase activity in control siRNA-treated DU145 cells (Figure 7B). Furthermore, we demonstrated that while SIPL1-C slightly reduced PTEN's PIP $_{3}$ phosphatase activity in the cell, SIPL1- $\triangle$ UBL was incapable of inhibiting PTEN's $\mathrm{PIP}_{3}$ phosphatase activity (Figure 7C). These observations are consistent with the results obtained by using the respective recombinant proteins (Figure 6). Taken together, the above observations clearly show that SIPL1 reduces PTEN's $\mathrm{PIP}_{3}$ phosphatase activity.

SIPL1 promotes tumorigenesis via inhibition of PTEN function. Our observations that SIPL1 inhibits PTEN's PIP 3 phosphatase activity and thus enhances the activities of the PI3K/AKT pathway suggest that SIPL1 promotes tumorigenesis. To investigate SIPL1-mediated tumorigenesis, we ectopically expressed SIPL1 in DU145 cells and subsequently implanted these cells s.c. into nude mice. In comparison to EV-transfected cells, DU145 cells infected with SIPL1 showed increased formation of xenograft tumors (Table 1). The enhancement of xenograft tumor formation by SIPL1 does not depend on specific cell lines or the immunocompromised animal host being used, as SIPL1 potently promoted the formation by HeLa cells of xenograft tumors in NOD/SCID mice (Figure 8A and Supplemental Figure 7A). As expected, xenograft tumors expressed PTEN, high levels of AKT activation, and ectopic SIPL1 (Supplemental Figure 7B). Since SIPL1-C is able to bind PTEN while SIPL1- $\triangle \mathrm{UBL}$ is incapable of binding PTEN, we also examined their impact on xenograft tumor formation. HeLa cells were infected with EV, SIPL1- $\triangle$ UBL, and SIPL1-C retrovirus (Supplemental Figure 8A) before being s.c. implanted into $\mathrm{NOD} / \mathrm{SCID}$ mice. In comparison to the EV cells, overexpression of either SIPL1- $\triangle$ UBL or SIPL1-C did not enhance the formation of xenograft tumors (Supplemental Table 1). This was not due to potential loss of the respective ectopic proteins, as xenograft tumors clearly expressed ectopic SIPL1- $\triangle$ UBL and SIPL1-C (Supplemental Figure 8B). These results are consistent with our observations that SIPL1- $\triangle$ UBL is incapable of interacting with PTEN (Figure 4, C and E) and that SIPL1-C's ability to reduce PTEN's PIP $_{3}$ phosphatase activity is substantially reduced compared with that of SIPL1 (Figures 6 and 7). Taken together, the above results suggest that full-length SIPL1 is required to enhance xenograft tumor formation.

We subsequently examined whether SIPL1 promotes the formation of xenograft tumors via inhibition of PTEN function. To address this issue, we overexpressed SIPL1 in the PTEN-nega- tive C33A cervical cancer cells (Figure 8C, inset) and implanted these cells into NOD/SCID mice. As expected, xenograft tumors expressed ectopic SIPL1 (data not shown). In comparison to EV cells, SIPL1 overexpression did not significantly enhance xenograft tumor formation (Figure $8 \mathrm{C}$ ). To further examine the role of endogenous SIPL1 in inhibiting endogenous PTEN function during tumor formation, we employed a system used by others to knock down SIPL1 in order to determine its impact on xenograft tumor formation (34). PTEN-positive HeLa cervical cancer cells were treated with combinations of control siRNA, SIPL1 siRNA, and PTEN siRNA oligonucleotides (Figure 8B, inset) for 48 hours. After confirmation of knockdown of individual proteins, cells were s.c. implanted into NOD/SCID mice. Xenograft tumor formation was significantly enhanced in PTEN-knockdown compared with control siRNA-treated cells (Figure 8B and Supplemental Table 2). Knockdown of SIPL1 dramatically inhibited the formation of HeLa cell-derived xenograft tumors (Figure 8B and Supplemental Table 2), which was largely reversed when PTEN was concomitantly knocked down (Figure 8B and Supplemental Table 2). These observations therefore support the notion that SIPL1 promotes the formation of xenograft tumors, at least in part, by inhibiting PTEN function.

To support the above concept, we knocked down SIPL1 in PTEN-null C33A cells (Supplemental Figure 9A, right panel). While xenograft tumors maintained SIPL1 knockdown (Supplemental Figure 9B), reduction of endogenous SIPL1 protein had no effect on the formation of C33A cell-derived xenograft tumors (Supplemental Figure 9A, left panel). Taken together, the above observations demonstrate that knockdown of SIPL1 attenuates xenograft tumor formation, at least in part, by releasing its inhibition of PTEN function.

To further demonstrate the impact of SIPL1 on the formation of HeLa cell-derived xenograft tumors, we first s.c. implanted HeLa cells into NOD/SCID mice. At day 7, a control siRNA or SIPL1 siRNA was directly delivered into xenograft tumors using AteloGene (see Methods for details). In comparison to control siRNA, SIPL1 siRNA reduced tumor incidence and tumor size (Table 2 and Supplemental Figure 7C).

Coexpression of SIPL1 in PTEN-positive primary cervical cancers. Our observations that SIPL1 promotes HeLa cervical cancer cells to form xenograft tumors via inhibition of PTEN function prompted us to examine the relationship between SIPL1 and PTEN in primary human cervical cancers. Cervical cancer tissue microarray (TMA) slides (US Biomax) contained 5 normal cervical tissues, 5 cancer-adjacent normal cervical tissues, and 94 cases of cervical carcinoma, with 84 cases being squamous cell carcinoma (Supplemental Table 3). Normal cervical tissues expressed lower levels of SIPL1 than carcinomas (Figure 9A). Consistent with previous reports $(21,22)$, we found that $69 \%$ (65 of 94 ) of cervical cancers expressed PTEN (Table 3). In PTEN-positive cervical carcinomas, $76.9 \%$ (50 of 65 ) were SIPL1 positive in comparison to $27.6 \%$ (8 of 29) of PTEN-negative cervical cancers showing SIPL1 positivity (Table 3), demonstrating that SIPL1 has a tendency to coexpress with PTEN in cervical cancers. This concept is supported by the significant positive correlation between PTEN and SIPL1 coexpression in cervical cancers (Table 3 ).

SIPL1 associates with reduction in PTEN function in primary cervical cancer. Activation of the PI3K/AKT pathway plays an important role during cervical tumorigenesis (27). Since approximately $85 \%$ of primary cervical cancers strongly express PTEN $(21,22)$, our 
A

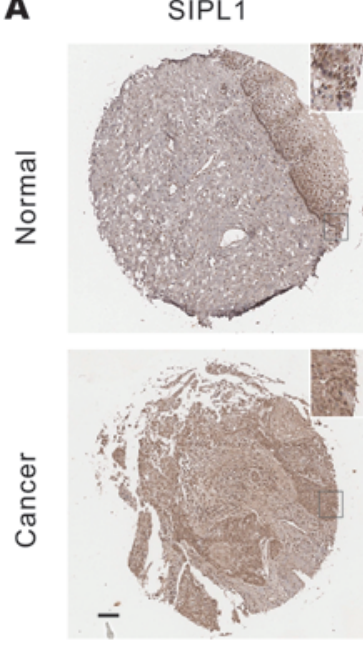

PTEN
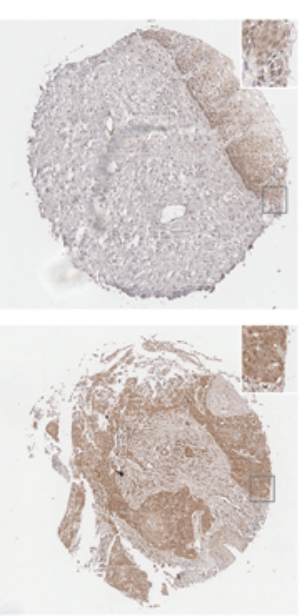

AKT-P
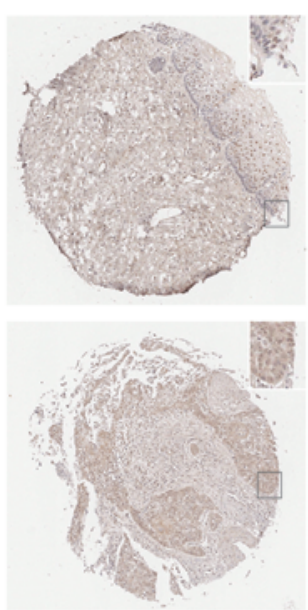
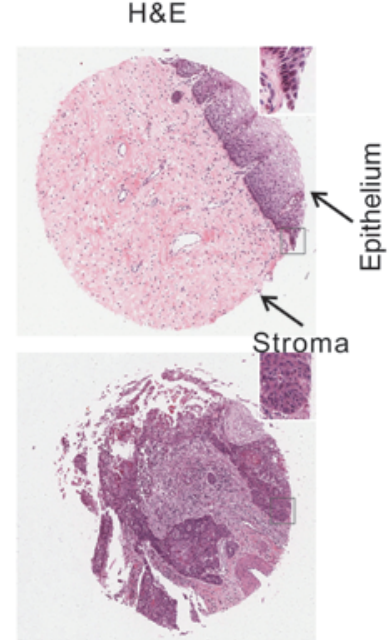

B
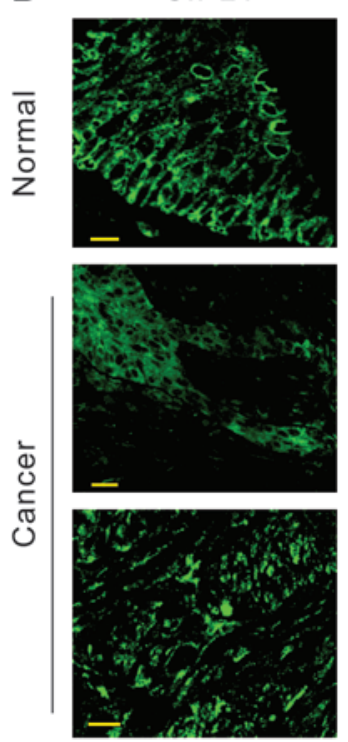

PTEN
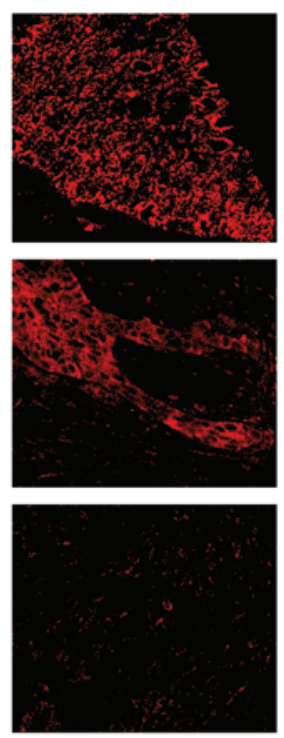

Merge
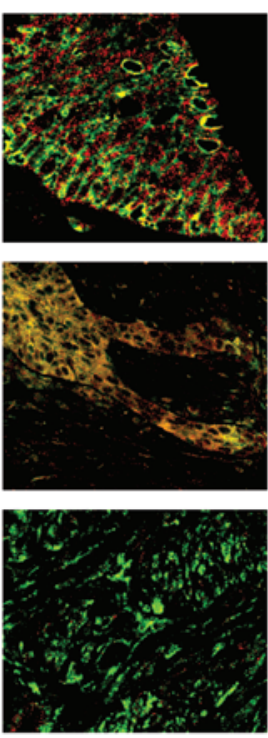
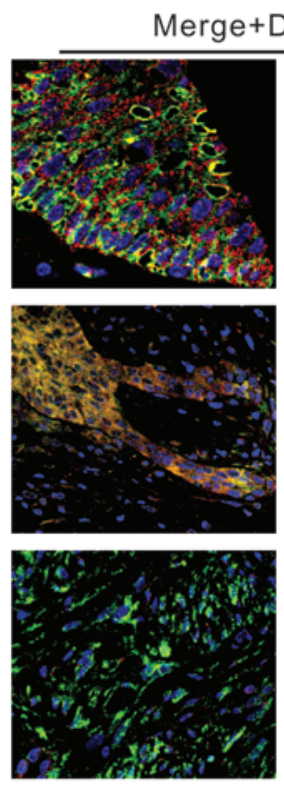
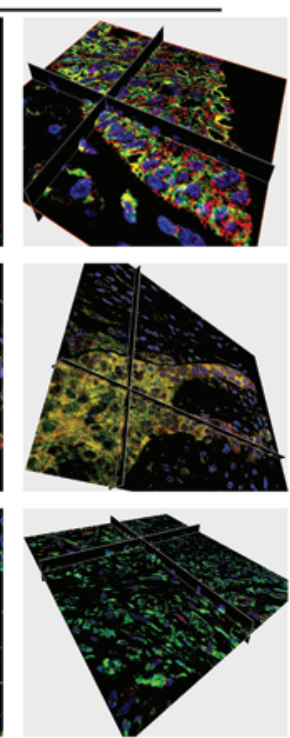

\section{Figure 9}

SIPL1 associates with loss of PTEN function in primary cervical cancer. (A) Cervical cancer tissue microarray slides were either H\&E or IHC stained with the indicated antibodies. Typical images are shown. The marked regions are enlarged 2.5-fold to show details (insets). Scale bar: $200 \mu \mathrm{m}$. (B) Cervical cancer tissue microarray slides were stained for SIPL1 (green) or PTEN (red) using indirect immunofluorescence. A set of z-stack images was acquired using an MP Leica TCS SP5 confocal microscope, and typical z-stack images are shown. Nuclei were counterstained with DAPI (blue). Three-dimensional images (right panels) constructed using Imaris software are also included. Scale bars: $10 \mu \mathrm{m}$ (top row), $20 \mu \mathrm{m}$ (bottom 2 rows).

observations that SIPL1 inhibits PTEN function, promotes formation of HeLa cervical cancer cell-derived xenograft tumors in immunocompromised mice by suppressing PTEN function, and coexists preferentially with PTEN in primary cervical cancers suggested the intriguing possibility that SIPL1 may repress PTEN function in PTEN-positive primary cervical cancers. To investigate this possibility, we examined AKT activation status in primary cervical cancers. While $34.5 \%$ (10 of 29) of PTEN-negative cervical cancers displayed AKT activation, 64.6\% (42 of 65) of PTEN-positive cervical cancers showed AKT activation (Table 3 ). In comparison to $20 \%$ (2 of 10) of PTEN-negative and AKT-P-positive cervical cancers being SIPL1 positive, 83.3\% (35 of 42) of PTEN-positive and AKT-P-positive cervical cancers were SIPL1 positive. These observations strongly suggest that SIPL1 plays a critical role in inhibiting PTEN function. Additionally, this concept is consistent with our observations that SIPL1 inhibits PTEN function in vitro and preferentially coexists with PTEN in cervical cancers.

To further support the concept that SIPL1 reduces PTEN function in primary cervical cancer, we examined the colocalization between PTEN and SIPL1 in primary cervical cancers. While SIPL1 weakly colocalizes with PTEN in normal cervical tissues (Figure 9B), SIPL1 extensively colocalizes with PTEN in PTEN-positive, but not PTEN-negative, cervical carcinoma (Figure 9B). Quantification of the colocalization between SIPL1 and PTEN using Imaris software revealed that $69 \%$ and $44 \%$ of PTEN colocalized with SIPL1 in cervical carcinoma and normal tissues, respectively (data not shown), 


\section{Table 3}

SIPL1 associates with loss of PTEN function in primary cervical cancer

\begin{tabular}{|c|c|c|c|c|}
\hline & & AKT-P-A & AKT-P+A & Total ${ }^{B}$ \\
\hline \multirow[t]{3}{*}{ PTEN $^{-}$} & SIPL1- & 13 & 8 & 21 \\
\hline & SIPL1+ & 6 & 2 & 8 \\
\hline & Total & 19 & 10 & 29 \\
\hline \multirow[t]{3}{*}{ PTEN+ } & SIPL1- & 8 & 7 & 15 \\
\hline & SIPL1+ & 15 & 35 & 50 \\
\hline & Total & 23 & 42 & 65 \\
\hline
\end{tabular}

\begin{abstract}
ANumber of patients with cervical carcinoma that is negative (AKT-P-) or positive (AKT-P+) for AKT activation. BTotal number of patients. Pearson's coefficient (Pearson's $r$ computed on two dichotomous variables) for the following correlations: PTEN versus SIPL1, $0.47(P<0.001)$; PTEN versus AKT-P, $0.28(P=0.007)$; and SIPL1 versus AKT-P, $0.22(P=0.036)$. The analysis was performed using SPSS 10.0 for Windows software.
\end{abstract}

with the Pearson's colocalization coefficiencies for this colocalization in carcinoma and normal tissues being 0.6 and 0.2 , respectively $(P<0.01)$ (data not shown).

\section{Discussion}

While expression of the PTEN gene is frequently repressed by genetic alterations and by hypermethylation of the PTEN promoter in human cancers $(4,14,15)$, inactivation of PTEN may also be mediated by putative PTEN-NRs. These PTEN-NRs may modify PTEN, resulting in loss of PTEN function. Casein kinase 2 (CK2) has been shown to phosphorylate PTEN at its C terminus, which contributes to PTEN inactivation in human $\mathrm{T}$ cell leukemia $(35,36)$. NEDD4-1 is capable of inducing PTEN degradation via PTEN ubiquitination and has been suggested to play a role in PTEN reduction in primary human cancers (37). PTEN-NRs may also reduce PTEN function by activation of downstream events of the PTEN pathway. Although DJ-1 does not interact with PTEN, it negatively regulates PTEN function via activation of AKT (38).

We report here that SIPL1 is a PTEN-NR. Sharpin/SIPL1 was detected in the postsynaptic density of excitatory synapses in the rat brain and in multiple tissues $(32,33)$. SIPL1 is highly conserved among mammals including humans, chimpanzees, dogs, rats, and mice (39), suggesting that SIPL1 has important physiological functions. Consistent with this notion, we demonstrate here that SIPL1 is a bona fide PTEN negative regulator and thereby facilitates cervical tumorigenesis via inhibition of PTEN function. As a sizable proportion of other types of cancers, including breast cancers, also express PTEN (28), whether SIPL1 may contribute to PTEN inactivation in these cancers is an area worthy of exploration. We have begun to explore this possibility and found that in 152 primary breast cancers examined, SIPL1 positively correlated with PTEN expression (Pearson's correlation coefficient [Pearson's $\phi]=0.43$, $P=0.01$; data not shown) and associates with reduced PTEN function in PTEN-positive breast cancer (data not shown). Although the number of PTEN-positive breast cancer cases examined was rather small to make a firm conclusion regarding the correlation between SIPL1 and PTEN in breast cancer, these observations nonetheless support the possibility that SIPL1 plays a role in PTEN inactivation in other human cancers.

Although we have demonstrated that SIPL1 promotes tumorigenesis by inhibiting, at least in part, PTEN function, we cannot exclude the possibility that SIPL1 may also modulate tumorigen- esis independently of PTEN. This is based on our observations that while knockdown of SIPL1 potently inhibits HeLa cell proliferation, co-knockdown of PTEN only partially reversed the inhibitory effect imposed by SIPL1 knockdown in vitro (Figure 1D). However, SIPL1 siRNA-mediated reduction of HeLa cell-derived xenograft tumor formation was largely reversed upon concomitant knockdown of PTEN (Figure 8B). Additionally, this potential PTEN-independent effect may be cell dependent, as SIPL1 siRNA-mediated inhibition of DU145 cell growth was largely reversed when endogenous PTEN was concomitantly knocked down (Figure 1C). Taken together, our results indicate that while SIPL1 may enhance tumorigenesis via PTEN-independent pathways, SIPL1 clearly promotes tumorigenesis by inhibiting PTEN function. It should be further emphasized that these two mechanisms may not be mutually exclusive.

The potential mechanisms by which SIPL1 reduces PTEN function are complex. Although we were able to demonstrate that SIPL1 inhibited PTEN's $\mathrm{PIP}_{3}$ phosphatase activity and that knockdown of SIPL1 reduced AKT activation in multiple cells (Figure 2), we did not observe significant increases in AKT activation upon overexpression of SIPL1 in either DU145 and HeLa cells (data not shown), suggesting that SIPL1 may require additional factors to modulate AKT activation. This may also be a cell line-dependent effect, as ectopic SIPL1 enhances AKT activation in PTEN-positive MCF7 cells (Supplemental Figure 5B). Further experiments will be needed to better define the molecular basis underlining this process.

The PI3K pathway plays essential roles in tumorigenesis by coordinating cell cycle progression, survival, and cytoskeletal organization (5). PTEN is the major mechanism that suppresses the PI3K pathway, as loss of PTEN activates the PI3K/AKT pathway in mice and in cells (40-43). Since attenuation of PTEN function is a major step during tumorigenesis and a sizable population of breast, prostate, and renal cell carcinomas express PTEN normally with elevated levels of AKT activation $(28,44)$, cancer cells may inactivate PTEN using PTEN negative regulatory mechanisms. We provide evidence that one of these putative mechanisms is SIPL1-mediated PTEN inactivation. This is consistent with several lines of evidence. The EST data bank from the Cancer Genome Anatomy Project revealed high levels of SIPL1 expression in cervical cancer, GI tract tumors, head and neck cancer, liver cancer, and other cancers (http://cgap.nci.nih.gov). The SIPL1 gene resides at 8q24.3 (http://www.ncbi.nlm.nih.gov/). Amplification of 8q24.3 occurs commonly in sporadic breast cancer (45) and squamous cell cervical carcinoma (46), two types of carcinomas that display a tendency toward SIPL1 expression with PTEN positivity. Increases in the copy number at the 8q24.3 locus were also observed in astrocytoma (47), gastroesophageal junction cancer (48), prostate cancer (49), chemoresistant ovarian cancer (50), larynx and pharynx squamous cell carcinoma (51), liver cancer (52, $53)$, and colorectal cancer $(54,55)$. Taken together, these observations suggest that amplification of SIPL1 facilitates the tumorigenesis of multiple human cancers. As inactivation of PTEN is widely regarded as a critical event during the tumorigenesis of all these cancers, amplification of SIPL1 may contribute to PTEN inactivation during tumorigenesis.

In addition to suppressing tumorigenesis, PTEN also plays essential roles in development and other physiological processes (56). It will thus be intriguing to examine whether SIPL1 also regulates PTEN function in these settings. It has been shown recently that two frameshift mutants in SIPL1 caused severe inflammation, impaired secondary lymphoid organ development, and induced 
dermatitis in mice (39). As observed in cancer, will any of these effects be caused by enhancing PTEN function as a consequence of loss of the PTEN negative regulator SIPL1?

\section{Methods}

Cell lines. Prostate cancer cell lines (DU145 and LNCaP), a glioma cell line (U87), cervical cancer cell lines (HeLa and C33A), breast cancer cell lines (MCF7 and HCC1954), 293 T cells (human 293 kidney embryonic epithelial cells expressing a temperature-sensitive SV40 T-antigen), human lung fibroblasts (IMR90), and mouse embryonic fibroblasts (NIH3T3) were obtained from ATCC and cultured according to the conditions specified. Immortalized and non-tumorigenic prostate epithelial cells (BPH-1) were provided by Simon W. Hayward of the Vanderbilt University Medical Center, Nashville, Tennessee, USA. 293T cells expressed very low levels of PTEN protein (data not shown). Both LNCaP and U87 cells are PTEN negative $(2,8,57)$. C33A cells did not express detectable PTEN protein (Supplemental Figure 10). The remaining cell lines expressed readily detectable PTEN protein.

Immunofluorescence and confocal microscopy. Double immunofluorescence staining was carried out using the following antibodies: monoclonal antiPTEN (1 $\mu \mathrm{g} / \mathrm{ml}$; Santa Cruz Biotechnology Inc.) or a polyclonal anti-PTEN (1:100; Upstate Technologies), polyclonal anti-FLAG or a monoclonal antiFLAG (M2, 1:500; Sigma-Aldrich). FITC-donkey anti-mouse IgG (1:200), rhodamine-donkey anti-rabbit IgG (1:200), and FITC-goat anti-mouse $\operatorname{IgM}$ (1:200; all Jackson ImmunoResearch Laboratories Inc.) were used as secondary antibodies. Images were captured using an MP Leica TCS SP5 confocal microscope. Images were analyzed using Imaris software (Bitplane) and processed using CorelDRAW 14 and Adobe Photoshop 7.

For dual immunofluorescence staining, TMA slides (US Biomax) were deparaffinized, rehydrated, and subjected to antigen retrieval and endogenous peroxidase quenching as indicated for IHC staining. Tissue sections were blocked for 1 hour at room temperature in 3\% donkey serum and 3\% BSA in TBST. Dual immunofluorescence staining was carried out using a TSA Plus kit (PerkinElmer) according to the manufacturer's protocol. Sections were counterstained with DAPI. Images were captured using an MP Leica TCS SP5 confocal microscope. Processing of images was performed using ImageJ bundled with the MBF "ImageJ for Microscopy" collection of plug-ins (http://www.macbiophotonics.ca/imagej/) and CorelDRAW 14.

Western blot and IP. Preparation of cell lysates and performance of Western blotting were carried out according to our published procedure (58). IP was carried out by incubation of cell lysates containing $200 \mu \mathrm{g}$ protein with specific antibodies plus Protein $\mathrm{G}$ agarose (Invitrogen) at $4^{\circ} \mathrm{C}$ overnight, followed by washing 6 times in a buffer containing $50 \mathrm{mM}$ Tris ( $\mathrm{pH} 7.5$ ), $100 \mathrm{mM} \mathrm{NaCl}, 1.5 \mathrm{mM}$ EGTA, 0.1\% Triton X-100. Antibodies used for IP were monoclonal anti-PTEN (Santa Cruz Biotechnology Inc.), monoclonal anti-HA (12CA5, Y-11, Santa Cruz Biotechnology Inc.), monoclonal antiFLAG (M2, Sigma-Aldrich) for SIPL1, polyclonal anti-SIPL1, and mouse as well as rabbit IgG (Sigma-Aldrich) as negative controls. The IP was analyzed by Western blot using polyclonal anti-PTEN (Santa Cruz Biotechnology Inc.), anti-FLAG (Sigma-Aldrich), and anti-SIPL1 antibodies. Quantification of protein bands detected by Western blot was carried out using Scion Image software (see Figure 2 legend for details).

Generation of anti-SIPL1 antibody. Recombinant GST-SIPL1 and 6xHistagged SIPL1 were isolated from E. coli BL-21 cells. Purified GST-SIPL1 was injected into 2 rabbits (8-10 weeks old). Rabbits were boosted with 6xHistagged SIPL1. The specific antibody was purified from rabbit serum by ammonium sulfate $\left[\left(\mathrm{NH}_{4}\right)_{2} \mathrm{SO}_{4}\right]$ precipitation, followed by passing through a GST-coupled CNBr-activated Sepharose 4B (Sigma-Aldrich) column to exclude IgG against GST. The flow-through was then applied to a GSTSIPL1-coupled Sepharose 4B column to purify the anti-SIPL1 antibody.
RNA interference. PTEN siRNA was expressed by a retroviral-based H1 promoter-driven shRNA vector ( $\mathrm{pRIH}$ ). The PTEN targeting sequence (GTATAGAGCGTGCAGATAA) was previously described (59). The SIPL1 siRNA target sequence was GAGTCAGTTTCCTACACCA, and its control siRNA sequence was generated by substitution of 4 nucleotides (indicated with underlines): GATTCAGTTGACTACAACA. Although the specificity of PTEN shRNA has been previously demonstrated (59), we still used a second PTEN siRNA sequence (TGTCTCTGGTCCTTACTTCTT). PTEN shRNA and PTEN siRNA produced the same results (data not shown). The specificity of SIPL1 siRNA was confirmed by two approaches: using a second siRNA (GGACAGAATGGCAGCAAGA) and an siRNA-resistant SIPL1 mutant (SIPL1*). The siRNA-resistant SIPL1* was generated by changing siRNA targeting nucleotide sequence but maintaining the amino acid residues [226-TCA(Ser)GTT(Val)TCC(Ser)-234 to 226-AGC(Ser)GTC(Val) 234; encoded amino acids are indicated in parentheses and substituted nucleotides are underlined] using site-directed mutagenesis $(60,61)$.

Knockdown of SIPL1 in xenograft tumors. AteloGene has been used to deliver siRNAs to xenograft tumors $(62-64)$. HeLa cells $\left(6 \times 10^{6}\right)$ were s.c. implanted into 10 nude mice. At day 7 , mice were randomly divided into two groups (5 mice per group), which were treated with either a control or SIPL1 siRNA using AteloGene (Koken) according the manufacturer's instructions. All animal protocols were approved by the Animal Research Ethics Board of McMaster University and were in compliance with institutional guidelines on the care of experimental animals.

PTEN lipid phosphatase assay. Purification of recombinant proteins (GST, GST-PTEN, GST-SIPL1, GST-SIPL1- $\triangle$ UBL, and GST-SIPL1-C) was performed in E. coli (BL21) as previously described (60). GST-PTEN (0.3 $\mu \mathrm{g})$ was incubated with GST (negative control), GST-SIPL1, or GST-SIPL1 mutants according to experimental design (see Figure 6 for details) for 30 minutes on ice, followed by the addition of $50 \mathrm{nM}$ water-soluble $\mathrm{DiC}_{8}$ PtdIns $(3,4,5) \mathrm{P}_{3}$ (Echelon) as a substrate to assay for $\mathrm{PIP}_{3}$ phosphatase activity at $37^{\circ} \mathrm{C}$ for 40 minutes. Released free phosphates were measured with BIOMOL Green reagent and were normalized against a reaction containing $\mathrm{PIP}_{3}$ substrate only (65).

To assay for cellular PTEN's PIP $_{3}$ phosphatase activity, PTEN and SIPL1 were cotransfected into 293T cells. Ectopic PTEN was immunoprecipitated with an anti-PTEN antibody (Santa Cruz Biotechnology Inc.), and subsequently assessed for phosphatase activity. Endogenous PTEN was also immunoprecipitated from DU145 cells or SIPL1-knockdown DU145 cells, followed by in vitro assay for PTEN's $\mathrm{PIP}_{3}$ phosphatase activity.

IHC staining of SIPL1, PTEN, and AKT activation in cervical cancer. TMA slides were deparaffinized and heat treated for 20 minutes in a $10-\mathrm{mM}$ sodium citrate buffer ( $\mathrm{pH}$ 6.0) in a food steamer. The following primary antibodies were incubated with the sections overnight at $4{ }^{\circ} \mathrm{C}$ : anti-SIPL1 (1:500), anti-PTEN (1:100; Santa Cruz Biotechnology Inc.), and anti-phospho-AKT (Ser473) (1:200; Cell Signaling Technology). Biotinylated goat anti-rabbit IgG and avidin-biotin complex (ABC) were then added (Vectastain ABC kit, Vector Laboratories). The chromogen reaction was carried out with diaminobenzidine, and counterstaining was done with hematoxylin. TMA slides were scanned using a ScanScope and analyzed using ImageScope software (Aperio). All spots (stained cores) were also manually examined. The scores obtained using the ImageScope software were converted to HScore using the formula [ $(\mathrm{HScore}=\%$ positive $\mathrm{X}$ (intensity +1)] $(66,67)$. Scores less than 260 and greater than 260 were assigned to negative (group 0 ) and positive (group 1) groups, respectively, which were used for statistical analysis (68).

Statistics. Statistical analysis was performed using SPSS 10.0 for Windows software. Data are presented as mean \pm SD unless otherwise specified. For immunohistochemical analysis, Pearson's $\phi$ coefficient (Pearson's $r$ computed on two dichotomous variables) was used to test for correlations between 
SIPL1 and PTEN, between SIPL1 and phospho-AKT (Ser473) (AKT-P), as well as between PTEN and AKT-P in cervical and breast cancers. All tests were 2 tailed. A $P$ value less than 0.05 was considered statistically significant.

\section{Acknowledgments}

We thank Tak Mak for providing HA-tagged PTEN and Richard Austin for reading the manuscript and for thoughtful discussions. The authors would also like to thank Lieqi Liu for superb technical assistance. L. He is supported by a postdoctoral fellowship from the Father Sean O'Sullivan Research Centre (FSORC). We also acknowledge financial support from St. Joseph's HealthCare, Hamilton,
Ontario, Canada, and the Hamilton Centre for Kidney Research (HCKR). This research was supported by grants from Prostate Cancer Canada and the Cancer Research Society to D. Tang.

Received for publication August 10, 2009, and accepted in revised form March 17, 2010.

Address correspondence to: Damu Tang, T3310, St. Joseph's Hospital, 50 Charlton Ave. East, Hamilton, Ontario, Canada L8N 4A6. Phone: 905.522.1155, ext. 35168; Fax: 905.540 .6549 or 905.521.6181; E-mail: damut@mcmaster.ca.
1. Myers MP, et al. P-TEN, the tumor suppressor from human chromosome $10 \mathrm{q} 23$, is a dualspecificity phosphatase. Proc Natl Acad Sci U S A. 1997;94(17):9052-9057.

2. Myers MP, et al. The lipid phosphatase activity of PTEN is critical for its tumor supressor function. Proc Natl Acad Sci U S A. 1998;95(23):13513-13518.

3. Maehama T, Dixon JE. The tumor suppressor, PTEN/MMAC1, dephosphorylates the lipid second messenger, phosphatidylinositol 3,4,5-trisphosphate. J Biol Chem. 1998;273(22):13375-13378.

4. Cantley LC, Neel BG. New insights into tumor suppression: PTEN suppresses tumor formation by restraining the phosphoinositide 3 kinase/AKT pathway. Proc Natl Acad Sci U S A. 1999;96(8):4240-4245.

5. Yuan TL, Cantley LC. PI3K pathway alterations in cancer; variations on a theme. Oncogene. 2008;27(41):5497-5510.

6. Li J, et al. PTEN, a putative protein tyrosine phosphatase gene mutated in human brain, breast, and prostate cancer. Science. 1997; 275(5308):1943-1947.

7. Steck PA, et al. Identification of a candidate tumour suppressor gene, MMAC1, at chromosome 10q23.3 that is mutated in multiple advanced cancers. Nat Genet. 1997;15(4):356-362.

8. Li DM, Sun H. PTEN/MMAC1/TEP1 suppresses the tumorigenicity and induces $\mathrm{G} 1$ cell cycle arrest in human glioblastoma cells. Proc Natl Acad Sci US A. 1998;95(26):15406-15411.

9. Bastola DR, Pahwa GS, Lin MF, Cheng PW. Downregulation of PTEN/MMAC/TEP1 expression in human prostate cancer cell line DU145 by growth stimuli. Mol Cell Biochem. 2002;236(1-2):75-81.

10. Di Cristofano A, Pandolfi PP. The multiple roles of PTEN in tumor suppression. Cell. 2000; 100(4):387-390.

11. Lee JO, et al. Crystal structure of the PTEN tumor suppressor: implications for its phosphoinositide phosphatase activity and membrane association. Cell. 1999;99(3):323-334.

12. Liaw D, et al. Germline mutations of the PTEN gene in Cowden disease, an inherited breast and thyroid cancer syndrome. Nat Genet. 1997;16(1):64-67.

13. Mallory SB. Cowden syndrome (multiple hamartoma syndrome). Dermatol Clin. 1995;13(1):27-31.

14. Goel A, et al. Frequent inactivation of PTEN by promoter hypermethylation in microsatellite instability-high sporadic colorectal cancers. Cancer Res. 2004;64(9):3014-3021.

15. Whang YE, et al. Inactivation of the tumor suppressor PTEN/MMAC1 in advanced human prostate cancer through loss of expression. Proc Natl Acad Sci US A. 1998;95(9):5246-5250.

16. Cheung TH, et al. Epigenetic and genetic alternation of PTEN in cervical neoplasm. Gynecol Oncol. 2004;93(3):621-627.

17. Su TH, et al. Mutation analysis of the putative tumor suppressor gene PTEN/MMAC1 in cervical cancer. Gynecol Oncol. 2000;76(2):193-199.

18. Yaginuma Y, et al. Abnormal structure and expression of PTEN/MMAC1 gene in human uterine cancers.
Mol Carcinog. 2000;27(2):110-116.

19. Harima Y, Sawada S, Nagata K, Sougawa M, Ostapenko V, Ohnishi T. Mutation of the PTEN gene in advanced cervical cancer correlated with tumor progression and poor outcome after radiotherapy. Int J Oncol. 2001;18(3):493-497.

20. Kurose K, Zhou XP, Araki T, Eng C. Biallelic inactivating mutations and an occult germline mutation of PTEN in primary cervical carcinomas. Genes Chromosomes Cancer. 2000;29(2):166-172.

21. El-Mansi MT, Williams AR. Evaluation of PTEN expression in cervical adenocarcinoma by tissue microarray. Int J Gynecol Cancer. 2006; 16(3):1254-1260.

22. Lee JS, et al. Expression of PTEN in the progression of cervical neoplasia and its relation to tumor behavior and angiogenesis in invasive squamous cell carcinoma. J Surg Oncol. 2006;93(3):233-240.

23. Beaudenon S, Huibregtse JM. HPV E6, E6AP and cervical cancer. BMC Biochem. 2008;9(suppl 1):S4.

24. Yugawa T, Kiyono T. Molecular mechanisms of cervical carcinogenesis by high-risk human papillomaviruses: novel functions of E6 and E7 oncoproteins. Rev Med Virol. 2009;19(2):97-113.

25. Contreras-Paredes A, De la Cruz-Hernández E, Martínez-Ramírez I, Dueñas- González A, Lizano M. E6 variants of human papillomavirus 18 differentially modulate the protein kinase B/phosphatidylinositol 3-kinase (akt/PI3K) signaling pathway. Virology. 2009;383(1):78-85.

26. Qi M, Anderson AE, Chen DZ, Sun S, Auborn KJ. Indole-3-carbinol prevents PTEN loss in cervical cancer in vivo. Mol Med. 2005;11(1-12):59-63.

27. Bertelsen BI, Steine SJ, Sandvei R, Molven A, Laerum OD. Molecular analysis of the PI3K-AKT pathway in uterine cervical neoplasia: frequent PIK3CA amplification and AKT phosphorylation. Int J Cancer. 2006;118(8):1877-1883.

28. Cully M, You H, Levine AJ, Mak TW. Beyond PTEN mutations: the PI3K pathway as an integrator of multiple inputs during tumorigenesis. Nat Rev Cancer. 2006;6(3):184-192.

29. Robertson GP, et al. In vitro loss of heterozygosity targets the PTEN/MMAC1 gene in melanoma. Proc Natl Acad Sci USA. 1998;95(16):9418-9423.

30. Furnari FB, Huang HJ, Cavenee WK. The phosphoinositol phosphatase activity of PTEN mediates a serum-sensitive $\mathrm{G} 1$ growth arrest in glioma cells. Cancer Res. 1998;58(22):5002-5008.

31. Franke TF, Kaplan DR, Cantley LC. PI3K: downstream AKTion blocks apoptosis. Cell. 1997;88(4):435-437.

32. Lim S, et al. Sharpin, a novel postsynaptic density protein that directly interacts with the shank family of proteins. Mol Cell Neurosci. 2001;17(2):385-397.

33. Daigo Y, Takayama I, Ward SM, Sanders KM, Fujino MA. Novel human and mouse genes encoding a shank-interacting protein and its upregulation in gastric fundus of W/WV mouse. J Gastroenterol Hepatol. 2003;18(6):712-718.

34. Junttila MR, et al. CIP2A inhibits PP2A in human malignancies. Cell. 2007;130(1):51-62.

35. Torres J, Pulido R. The tumor suppressor PTEN is phosphorylated by the protein kinase CK2 at its C terminus. Implications for PTEN stability to proteasome-mediated degradation. J Biol Chem. 2001;276(2):993-998.

36. Silva A, et al. PTEN posttranslational inactivation and hyperactivation of the PI3K/Akt pathway sustain primary $\mathrm{T}$ cell leukemia viability. J Clin Invest. 2008;118(11):3762-74.

37. Wang $X$, et al. NEDD4-1 is a proto-oncogenic ubiquitin ligase for PTEN. Cell. 2007;128(1):129-139.

38. Kim RH, et al. DJ-1, a novel regulator of the tumor suppressor PTEN. Cancer Cell. 2005;7(3):263-273.

39. Seymour RE, et al. Spontaneous mutations in the mouse Sharpin gene result in multiorgan inflammation, immune system dysregulation and dermatitis. Genes Immun. 2007;8(5):416-21.

40. Di Cristofano A, Pesce B, Cordon-Cardo C, Pandolfi PP. Pten is essential for embryonic development and tumour suppression. Nat Genet. 1998;19(4):348-355.

41. Suzuki A, et al. High cancer susceptibility and embryonic lethality associated with mutation of the PTEN tumor suppressor gene in mice. Curr Biol. 1998;8(21):1169-1178.

42. Stambolic V, et al. Negative regulation of PKB/Aktdependent cell survival by the tumor suppressor PTEN. Cell. 1998;95(1):29-39.

43. Haas-Kogan D, Shalev N, Wong M, Mills G, Yount $\mathrm{G}$, Stokoe D. Protein kinase B (PKB/Akt) activity is elevated in glioblastoma cells due to mutation of the tumor suppressor PTEN/MMAC. Curr Biol. 1998;8(21):1195-1198.

44. He L, et al. Co-existence of high levels of the PTEN protein with enhanced Akt activation in renal cell carcinoma. Biochim Biophys Acta. 2007;1772(10):1134-1142.

45. Naylor TL, et al. High resolution genomic analysis of sporadic breast cancer using array-based comparative genomic hybridization. Breast Cancer Res. 2005;7(6):R1186-R1198.

46. Choi YW, et al. Gene expression profiles in squamous cell cervical carcinoma using array-based comparative genomic hybridization analysis. Int $J$ Gynecol Cancer. 2007;17(3):687-696.

47. Jeon YK, Cheon JE, Kim SK, Wang KC, Cho BK, Park $\mathrm{SH}$. Clinicopathological features and global genomic copy number alterations of pilomyxoid astrocytoma in the hypothalamus/optic pathway: comparative analysis with pilocytic astrocytoma using array-based comparative genomic hybridization. Mod Pathol. 2008;21(11):1345-56.

48. van Duin M, et al. High-resolution array comparative genomic hybridization of chromosome 8q: evaluation of putative progression markers for gastroesophageal junction adenocarcinomas. Cytogenet Genome Res. 2007;118(2-4):130-137.

49. $\mathrm{Kim} \mathrm{JH}$, et al. Integrative analysis of genomic aberrations associated with prostate cancer progression. Cancer Res. 2007;67(17):8229-8239.

50. Kim SW, et al. Analysis of chromosomal changes in serous ovarian carcinoma using high- resolution array comparative genomic hybridization: Potential predictive markers of chemoresistant disease. Genes 
Chromosomes Cancer. 2007;46(1):1-9.

51. Hermsen $\mathrm{M}$, et al. Chromosomal changes in relation to clinical outcome in larynx and pharynx squamous cell carcinoma. Cell Oncol. 2005;27(3):191-198.

52. Okamoto H, Yasui K, Zhao C, Arii S, Inazawa J. PTK2 and EIF3S3 genes may be amplification targets at 8q23-q24 and are associated with large hepatocellular carcinomas. Hepatology. 2003;38(5):1242-1249.

53. Chochi $Y$, et al. A copy number gain of the $6 \mathrm{p}$ arm is linked with advanced hepatocellular carcinoma: an array-based comparative genomic hybridization study. J Pathol. 2009;217(5):677-684.

54. Douglas EJ, et al. Array comparative genomic hybridization analysis of colorectal cancer cell lines and primary carcinomas. Cancer Res. 2004;64(14):4817-4825.

55. Buffart TE, et al. DNA copy number changes at 8q11-24 in metastasized colorectal cancer. Cell Oncol. 2005;27(1):57-65.

56. Suzuki A, Nakano T, Mak TW, Sasaki T. Portrait of PTEN: messages from mutant mice. Cancer Sci. 2008;99(2):209-213.

57. Davies MA, et al. Regulation of Akt/PKB activ- ity, cellular growth, and apoptosis in prostate carcinoma cells by MMAC/PTEN. Cancer Res. 1999;59(11):2551-2556

58. Tang $\mathrm{D}$, et al. Akt is activated in response to an apoptotic signal. J Biol Chem. 2001;276(32):30461-30466.

59. Tang X, Powelka AM, Soriano NA, Czech MP, Guilherme A. PTEN, but not SHIP2, suppresses insulin signaling through the phosphatidylinositol 3- kinase/Akt pathway in 3T3-L1 adipocytes. J Biol Chem. 2005;280(23):22523-22529.

60. Tang D, Chun AC, Zhang M, Wang JH. Cyclindependent kinase 5 (Cdk5) activation domain of neuronal Cdk5 activator. Evidence of the existence of cyclin fold in neuronal Cdk5a activator. J Biol Chem. 1997;272(19):12318-12327.

61. Tang D, Gururajan R, Kidd VJ. Phosphorylation of PITSLRE p110 isoforms accompanies their processing by caspases during Fas-mediated cell death. J Biol Chem. 1998;273(26):16601-16607.

62. Ochiya $\mathrm{T}$, et al. New delivery system for plasmid DNA in vivo using atelocollagen as a carrier material: the Minipellet. Nat Med. 1999;5(6):707-710.

63. Yu D, Sekine E, Fujimori A, Ochiya T, Okayasu R
Down regulation of BRCA2 causes radio-sensitization of human tumor cells in vitro and in vivo. Cancer Sci. 2008;99(4):810-815.

64. Iwaki $\mathrm{K}$, et al. A small interfering RNA targeting proteinase-activated receptor-2 is effective in suppression of tumor growth in a Panc1 xenograft model. Int J Cancer. 2008;122(3):658-663.

65. Georgescu MM, Kirsch KH, Akagi T, Shishido T, Hanafusa $H$. The tumor-suppressor activity of PTEN is regulated by its carboxyl-terminal region. Proc Natl Acad Sci U S A. 1999;96(18):10182-10187.

66. Randall LM, et al. Markers of angiogenesis in high-risk, early-stage cervical cancer: A Gynecologic Oncology Group study. Gynecol Oncol. 2009;112(3):583-589.

67. Mehta R, et al. Independent association of angiogenesis index with outcome in prostate cancer. Clin Cancer Res. 2001;7(1):81-88.

68. Maw MK, Fujimoto J, Tamaya T. Expression of the inhibitor of DNA- binding (ID)-1 protein as an angiogenic mediator in tumour advancement of uterine cervical cancers. Br J Cancer. 2008;99(10):1557-1563. 